\title{
COMPUTING MINIMAL POLYNOMIALS OF MATRICES
}

\author{
MAX NEUNHÖFFER AND CHERYL E. PRAEGER
}

\begin{abstract}
We present and analyse a Monte-Carlo algorithm to compute the minimal polynomial of an $n \times n$ matrix over a finite field that requires $O\left(n^{3}\right)$ field operations and $O(n)$ random vectors, and is well suited for successful practical implementation. The algorithm, and its complexity analysis, use standard algorithms for polynomial and matrix operations. We compare features of the algorithm with several other algorithms in the literature. In addition we present a deterministic verification procedure which is similarly efficient in most cases but has a worst-case complexity of $O\left(n^{4}\right)$. Finally, we report the results of practical experiments with an implementation of our algorithms in comparison with the current algorithms in the GAP library.
\end{abstract}

\section{Introduction}

Let $\mathbb{F}$ be a finite field and $M \in \mathbb{F}^{n \times n}$ a matrix. This paper presents and analyses a Monte Carlo algorithm to compute the minimal polynomial of $M$, that is, the monic polynomial $\mu \in \mathbb{F}[x]$ of least degree, such that $\mu(M)=0$. Determining the minimal polynomial is one of the fundamental computational problems for matrices and has a wide range of applications. As well as revealing information about the Frobenius normal form of $M$, the minimal polynomial also elucidates the structure of $\mathbb{F}^{n}$ viewed as $\mathbb{F}[x]$-module, where $x$ acts by multiplication with $M$. In addition the order of $M$ modulo scalars is often found by first determining the minimal polynomial. Apart from these applications it has important practical utility, for example in the context of the matrix group recognition project [10].

For these and other reasons a number of algorithms to determine the minimal polynomial may be found in the literature. We discuss some of them below. Our primary objective was to provide a simple and practical algorithm that could be implemented easily and would work well over small finite fields. In particular we did not want to produce matrices with entries in larger fields or polynomial rings as intermediate results, and we preferred to restrict ourselves to using only row operations (rather than a combination of row and column operations). In addition we wished to use standard field and polynomial arithmetic, and we wished to give an explicit worst-case upper bound for the number of elementary field operations needed, and not only an asymptotic complexity statement. Our Monte Carlo algorithm adheres to these requirements for matrices over fields $\mathbb{F}_{q}$ of order $q$.

Received 8 May 2007, revised 5 February 2008; published 4 August 2008.

2000 Mathematics Subject Classification 15A21 (primary), 15A15 (secondary)

(C) 2008, Max Neunhöffer and Cheryl E. Praeger 
THEOREM 1.1. For a given matrix $M \in \mathbb{F}_{q}^{n \times n}$ and a positive real number $\varepsilon<$ 1/2, Algorithm 5 computes the minimal polynomial of $M$ with probability at least $1-\varepsilon$. For sufficiently large $n$ and fixed $\varepsilon$, the number of elementary field operations required is less than $7 n^{3}$ plus the costs of factorising a degree $n$ polynomial over $\mathbb{F}_{q}$ and constructing at most $n$ random vectors in $\mathbb{F}^{n}$.

Our algorithm to compute the minimal polynomial first computes the characteristic polynomial in a standard way by spinning up and then factoring out cyclic subspaces. However, the novel aspect in this first phase is the introduction of randomisation. While not necessary for the computation of the characteristic polynomial it underpins our proof of the Monte Carlo nature of our minimal polynomial algorithm. In addition to the Monte Carlo minimal polynomial algorithm we present and analyse in Section 8 a deterministic verification procedure to be run after Algorithm 5 that has a similar asymptotic complexity in many cases, but is $O\left(n^{4}\right)$ in the worst-case scenario. Our motivation for giving concrete upper bounds for the costs of various component procedures was that, in practical implementations, these assist us to compare different algorithms in order to decide which to use in different situations. At the end of the paper we discuss a practical implementation and tests of the algorithms in the GAP system [4].

\subsection{Other algorithms in the light of our requirements}

There are several interesting and asymptotically efficient minimal polynomial algorithms for $n \times n$ matrices in the literature. The most asymptotically efficient deterministic algorithm is due to Storjohann [14] in 2001. It is nearly optimal, 'requiring about the same number of field operations as required for matrix multiplication' (see [14, Abstract, p. 368]). It involves a divide-and-conquer strategy that produces matrices with entries in polynomial rings as intermediate results. Changing the scalars to a larger field or polynomial ring is something we wished to avoid as it creates additional complications in practical applications within a computer algebra system used for group and matrix algebra computations.

Storjohann's earlier deterministic algorithm [13] in 1998 uses classical field arithmetic and requires $O\left(n^{3}\right)$ field operations. It first reduces the matrix to 'zig-zag form', using a mix of row and column operations, then produces the Smith normal form as a matrix with polynomial entries, and finally the Frobenius normal form. In systems such as GAP, matrices over small finite fields are stored in a compressed form that makes row operations simple, but column operations difficult. Restricting to one of these types of operations was one of our criteria.

A Monte Carlo minimal polynomial algorithm of Giesbrecht [5] from 1995 that runs in 'nearly optimal time' contains some features we find desirable for practical implementation, namely his algorithm first constructs a 'modular cyclic decomposition' using random vectors, similar to our characteristic polynomial computation in Section 5. However, further steps include a modification of the 'divide-and-conquer' Keller-Gehrig algorithm [8] and lead to a Las Vegas algorithm that computes a Frobenius form over an extension field and then the minimal polynomial. The field size over which the given matrix is written is assumed to be greater than $n^{2}$, and if this is not the case it is suggested that an embedding into a larger extension field be used. Several of these features were undesirable for us. 
In [1, Section 4] Augot and Camion propose a deterministic algorithm to compute the minimal polynomial of a matrix which is to some extent similar to our algorithm. It is deterministic with complexity $O\left(n^{3}+m^{2} \cdot n^{2}\right)$ field operations, where $m$ is the number of blocks in the shift Hessenberg form. They prove that the complexity is $O\left(n^{3}\right)$ in the average case. However, in the worst case it is $O\left(n^{4}\right)$, and no constants are provided in the complexity estimates. Although the principal approach of their algorithm is similar to ours, the details differ very much from our algorithm and analysis.

An interesting commentary on various algorithms, together with some new algorithms is given by Eberly [3]. Eberly (see Theorem 4.2 in [3]) gives in particular a randomised algorithm for matrices over small fields that produces output from which (amongst other things) the minimal polynomial can be computed, at a cost of $O\left(n^{3}\right)$. The papers $[\mathbf{3}, \mathbf{5}, \mathbf{1 2}, \mathbf{1 3}, \mathbf{1 4}]$ contain references to other minimal polynomial algorithms. In all of the algorithms mentioned the asymptotic complexity statements give no information about the constants involved.

On a practical note, the minimal polynomial algorithm implemented in the GAP library is the one in [12] and (although we have been unable to confirm this) we assume that this is the algorithm implemented in Magma [2].

\subsection{Outline of the paper}

In Section 2 we introduce our notation, in Section 3 we cite a few complexity bounds for basic algorithms. The next Section 4 introduces order polynomials and derives a few results about them. Then we turn to the computation of the characteristic polynomial in Section 5, since this is the first step in our minimal polynomial algorithm, which is described and analysed in Section 7. We explain and modify the well-known algorithm to compute characteristic polynomials by introducing some randomisation, because this is later needed in the analysis of our main Monte Carlo algorithm. In Section 6 we give some probability estimates that are also used later in the analysis. The second last Section 8 covers the deterministic verification of the results of our Monte Carlo algorithm. We describe in detail cases in which this verification is efficient and when it has a worse complexity. Finally, in Section 9 we report on the performance of an implementation of our algorithm, including runtimes in realistic applications. We compare these times with the current implementation for minimal polynomial computations in the GAP library (see [4]), and as mentioned above, we believe that Magma and GAP are both using the algorithm in [12]. We show that our algorithm performs much better in important cases, and that our bounds on the computing cost are reflected in practical experiments.

\section{Notation}

Throughout the paper $\mathbb{F}$ will be a fixed field. Although we envisage $\mathbb{F}$ to be a finite field for our applications, this is not necessary for most of our results. However in the later sections we use some probability estimates from Section 6 that are only valid for finite fields.

By an elementary field operation we mean addition, subtraction, multiplication or division of two field elements. In all our runtime bounds we will assume that one elementary field operation takes a fixed amount of time and we simply count the number of such operations occurring in our algorithms. 
We denote the set of $(m \times n)$-matrices over $\mathbb{F}$ by $\mathbb{F}^{m \times n}$ and the set of row vectors of length $m$ by $\mathbb{F}^{m}$. For a vector $v \in \mathbb{F}^{m}$ we write $v_{i}$ for its $i$-th component and for a matrix $M \in \mathbb{F}^{m \times n}$ we denote its $i$-th row, which is a row vector of length $n$, by $M[i]$. We use "row vector times matrix" operations, and in general right modules throughout. If $V$ is a vector space over $\mathbb{F}$ and $W$ is a subspace, the quotient space is denoted by $V / W$ and its cosets by $v+W$ for $v \in V$. The $\mathbb{F}$-linear span of the vectors $v^{(1)}, \ldots, v^{(k)} \in V$ is denoted by $\left\langle v^{(1)}, \ldots, v^{(k)}\right\rangle_{\mathbb{F}}$.

If $M \in \mathbb{F}^{n \times n}$ is a matrix and $V=\mathbb{F}^{n}$, we have a natural action of $M$ as an endomorphism of $V$ by right multiplication. The same holds for every $M$-invariant subspace $W<V$ and for the corresponding quotient space $V / W$. We describe such a situation by saying that "the matrix $M$ induces an action on the $\mathbb{F}$-vector space" $V, W, V / W$ respectively.

Throughout, $\mathbb{F}[x]$ denotes the polynomial ring over $\mathbb{F}$ in an indeterminate $x$. For a square matrix $M$ and a polynomial $p \in \mathbb{F}[x]$ we denote the evaluation of $p$ at $M$ by $p(M)$.

Whenever a matrix $M$ induces an action on a vector space $U$, we will view $U$ as a right $\mathbb{F}[x]$-module by letting $x$ act like $M$, that is $v \cdot x:=v \cdot M$ in the above examples. We denote the characteristic polynomial of this action by $\chi_{M, U}$. That is, $\chi_{M, U}$ is the characteristic polynomial of the $\left(\operatorname{dim}_{\mathbb{F}}(U) \times \operatorname{dim}_{\mathbb{F}}(U)\right)$-matrix given by choosing a basis of $U$ and writing the action of $M$ induced on $U$ as a matrix with respect to that basis. We use the same convention analogously for the corresponding minimal polynomial $\mu_{M, U}$. Furthermore, we denote the $\mathbb{F}[x]$-submodule of $U$ generated by the vectors $u^{(1)}, \ldots, u^{(n)}$ by $\left\langle u^{(1)}, \ldots, u^{(n)}\right\rangle_{M}$.

We use the two functions

$$
s^{(1)}(a, b):=\sum_{i=b+1}^{a} i \text { and } s^{(2)}(a, b):=\sum_{i=b+1}^{a} i^{2}
$$

for complexity expressions. Note that for $a>b>c$ we have $s^{(j)}(a, c)=s^{(j)}(a, b)+$ $s^{(j)}(b, c)$ for $j \in 1,2$ and

$$
\begin{aligned}
& s^{(1)}(n, 0)=s^{(1)}(n,-1)=\frac{n(n+1)}{2} \text { and } \\
& s^{(2)}(n, 0)=s^{(2)}(n,-1)=\frac{n(n+1)(2 n+1)}{6} .
\end{aligned}
$$

For later complexity estimates we note the following inequalities.

Lemma 2.1 (Some upper bounds). If $n=\sum_{i=1}^{k} d_{i}$ for some $d_{i} \in \mathbb{N} \backslash\{0\}$ and $s_{j}:=\sum_{i=1}^{j} d_{i}$ we have

$$
\sum_{j=1}^{k} s_{j} \leqslant \frac{n(n+1)}{2} \quad \text { and } \quad \sum_{j=1}^{k} s_{j}\left(s_{j}+1\right) \leqslant \frac{n(n+1)(n+2)}{3} .
$$

Proof. We claim that for fixed $n$ both expressions are maximal if and only if all $d_{i}$ are equal to one. We leave it to the reader to check that both totals increase if we replace $d_{j}$ in some sequence $\left(d_{i}\right)_{1 \leqslant i \leqslant k}$ by the two numbers $a$ and $d_{j}-a$ resulting in the new sequence $\left(d_{1}^{\prime}, \ldots, d_{k+1}^{\prime}\right):=\left(d_{1}, d_{2}, \ldots, d_{j-1}, a, d_{j}-a, d_{j+1}, \ldots, d_{k}\right)$ of length $k+1$. 


\section{Complexity bounds for basic algorithms}

In some algorithms presented in later sections we use greatest common divisors of univariate polynomials. To analyse these algorithms we use the following bounds which arise from standard polynomial computation. We take this approach because the standard algorithms for polynomials are good enough for our complexity estimates in applications and we do not need the asymptotically best algorithms, discussion of which may be found conveniently in [15].

Proposition 3.1 (Complexity of standard greatest common divisor algorithm).

Let $f, g \in \mathbb{F}[x]$ with $n:=\operatorname{deg} f \geqslant \operatorname{deg} g=: m$, and $f=q g+r$ with $q, r \in \mathbb{F}[x]$ such that $r=0$ or $\operatorname{deg} r<\operatorname{deg} g$. Then there is an algorithm to compute $q$ and $r$ that needs less than $2(m+1)(n-m+1)$ elementary field operations.

Furthermore, there is an algorithm to compute $\operatorname{gcd}(f, g)$ that needs less than $2(m+1)(n+1)$ elementary field operations.

REMARK 3.2. We intentionally give bounds here which are not best possible, since we want the bound for the gcd computation to be symmetrical in $m$ and $n$.

Proof of Proposition 3.1. Use polynomial division and the standard GCD algorithm and count. See [15, Section 2.4 and Section 3.3] for smaller bounds that imply our symmetric bounds.

\subsection{Polynomial factorisation}

Some of our algorithms return partially factorised polynomials which facilitate later factorisation into irreducible factors. However, since the extent of this partial factorisation is difficult to estimate, we use in our analyses the complexity of finding the complete factorisation of a polynomial over a finite field as a product of irreducibles. We need such factorisations in our main algorithm. In keeping with our other methods we make use of standard polynomial factorisation procedures.

Details can be found in Knuth $[\mathbf{9}, 4.6 .2]$ of a deterministic polynomial factorisation algorithm inspired by an idea of Berlekamp. Its cost is polynomial in both the degree $n$ and field size $|\mathbb{F}|=q$, as it requires $O(q)$ computations of greatest common divisors. Thus it works well only for $q$ small. There is available a randomised (Las Vegas) version of the procedure which (for arbitrary $q$ ) will always return accurately the number $r$ of irreducible factors of $f(x) \in \mathbb{F}[x]$, but for which there is a small non-zero probability that it will fail to find all the irreducible factors. It involves the procedure RANDOMVECTOR, which is discussed further in Subsection 5.1, to produce independent uniformly distributed random elements of an $n$-dimensional vector space over $\mathbb{F}$ for which a basis is known. Throughout the paper logarithms are always taken to base 2 .

Remark 3.3 (PolynomialFactorisation). Suppose $f(x) \in \mathbb{F}[x]$ of degree $n \geqslant 1$ with $r$ irreducible factors (counting multiplicities) and, if $q$ is large, suppose that we are given a real number $\varepsilon$ such that $0<\varepsilon<1 / 2$. The number $\operatorname{fact}(n, q)$ of elementary field operations required to find a complete set of irreducible factors of $f(x)$ is at most

$$
\begin{array}{ll}
8 n^{3}+(3 q r+17 \log q) n^{2} & \text { deterministic algorithm } \\
O\left(\left(\log \varepsilon^{-1}\right)(\log n)\left(\xi_{n}+n^{2} \log ^{3} q\right)+n^{3} \log ^{2} q\right) & \text { Las Vegas algorithm }
\end{array}
$$


where $\xi_{n}$ is an upper bound for the cost of one run of RANDOMVECTOR on $\mathbb{F}^{n}$. The Las Vegas algorithm may fail, but with probability less than $\varepsilon$.

\section{Order polynomials}

Let $M$ be a matrix in $\mathbb{F}^{n \times n}$ that induces an action on an $\mathbb{F}$-vector space $V$. We briefly recall the definition of the term "order polynomial":

DEFinition 4.1 (Order polynomial $\operatorname{ord}_{M}(v)$ and relative order polynomial). The order polynomial ord $\cos _{M}(v)$ of a vector $v \in V$ is the monic polynomial $p \in \mathbb{F}[x]$ of smallest degree such that $v \cdot p(M)=0 \in V$. In particular $\operatorname{ord}_{M}(0)=1$.

For an $M$-invariant subspace $W<V$, the relative order polynomial $\operatorname{ord}_{M}(v+W)$ (of $v$ relative to $W$ ) is the order polynomial of the element $v+W \in V / W$ with respect to the induced action of $M$ on $V / W$.

REMARK 4.2. If we consider $V$ as an $\mathbb{F}[x]$-module as in Section 2 , then $p$ is the monic generator of the annihilator $\operatorname{ann}_{\mathbb{F}[x]}(v)$ of $v$ in $\mathbb{F}[x]$.

The following observation follows immediately from the definition above.

Lemma 4.3 (Relative order polynomials). For an $M$-invariant subspace $W<V$ and $v \in V, \operatorname{ord}_{M}(v+W)$ is the monic polynomial $p \in \mathbb{F}[x]$ of smallest degree such that $v \cdot p(M) \in W$.

We now turn to the question of how one computes the order polynomial of a vector $v \in V$. The basic idea is to apply the matrix $M$ to the vector repeatedly computing a sequence $v, v M, v M^{2}, \ldots, v M^{d}$ until $v M^{d}$ is a linear combination

$$
v M^{d}=\sum_{i=0}^{d-1} a_{i} v M^{i},
$$

with $a_{i} \in \mathbb{F}$, for $0 \leqslant i<d$. If $d$ is minimal such that this is possible, we have

$$
\operatorname{ord}_{M}(v)=x^{d}-\sum_{i=0}^{d-1} a_{i} x^{i} .
$$

Although this procedure is simple and well-known, we present it in order to make explicit the number of elementary field operations needed. To this end we describe in detail the computation of solutions for the systems of linear equations involved.

DEFinition 4.4 (Row semi echelon form). A non-zero matrix $S=\left(S_{i, j}\right) \in \mathbb{F}^{m \times n}$ is in row semi echelon form if there are positive integers $r \leqslant m$ and $j_{1}, \ldots, j_{r} \leqslant n$ such that, for each $i \leqslant r, S_{i, j_{i}}=1$ and $S_{k, j_{i}}=0$ for all $k>i$, and also $S_{k, j}=0$ whenever $k>r$. For $i \leqslant r$, column $j_{i}$ is called the leading column of row $i$, and we write $\operatorname{lc}(i)=j_{i}$. A sequence of vectors $u^{(1)}, \ldots, u^{(m)} \in F^{n}$ is said to be in semi echelon form if the matrix with rows $u^{(1)}, \ldots, u^{(m)}$ is in row semi echelon form.

Note that in Definition 4.4 we do not assume $j_{1}<j_{2}<\cdots<j_{r}$ which is the usual condition for an echelon form.

Definition 4.5 (Semi echelon data sequence). Let $Y \in \mathbb{F}^{m \times n}$ be a matrix with $m \leqslant n$ and of rank $m$. A semi echelon data sequence for $Y$ is a tuple $\mathcal{Y}=(Y, S, T, l)$, 
where $S \in \mathbb{F}^{m \times n}$ is in row semi echelon form with leading column indices $l=$ (lc $(1), \ldots, \operatorname{lc}(m))$, and $T \in \mathrm{GL}(m, \mathbb{F})$ with $T Y=S$. Further, $T$ is a lower triangular matrix, that is, for $T=\left(T_{i, j}\right)$ we have $T_{i, j}=0$ for $i<j$. For a semi echelon data sequence $\mathcal{Y}$ we call the number $m$ its length, sometimes denoted length $(\mathcal{Y})$. A semi echelon data sequence $\mathcal{Y}^{\prime}=\left(Y^{\prime}, S^{\prime}, T^{\prime}, l^{\prime}\right)$ is said to extend $\mathcal{Y}$ if length $\left(\mathcal{Y}^{\prime}\right)>\operatorname{length}(\mathcal{Y})$, the first length $(\mathcal{Y})$ rows of $Y^{\prime}$ and $S^{\prime}$ form the matrices $Y$ and $S$ respectively, and the first length $(\mathcal{Y})$ entries of $l^{\prime}$ form the sequence $l$.

REMARK 4.6. (a) The idea of this concept is that for a matrix $S \in \mathbb{F}^{m \times n}$ in row semi echelon form it is relatively cheap to decide whether a given vector $v \in \mathbb{F}^{n}$ lies in the row space of $S$, and if so, to write it as a linear combination of the rows of $S$, that is, to find a vector $a \in \mathbb{F}^{m}$ such that $v=a S=a T Y$ (see Algorithm 1). Thus, the vector $v$ is expressed as a linear combination of the rows of $Y$ using the vector $a T$ as coefficients.

(b) We call a semi echelon data sequence trivial if $m=0$. In this case, by convention, we take the row spaces of the empty matrices $Y$ and $S$ to be the zero subspace of $\mathbb{F}^{n}$, we denote the empty sequence in $\mathbb{F}^{0}$ by 0 , and we interpret $a S$ as the zero vector of $\mathbb{F}^{n}$.

We now present Algorithm 1, which is one step in the computation of a semi echelon data sequence for a matrix $Y$. We denote by $S[i]$ the $i$-th row of the matrix $S$, and by $\operatorname{Row} \operatorname{Sp}(S)$ the row space of $S$.

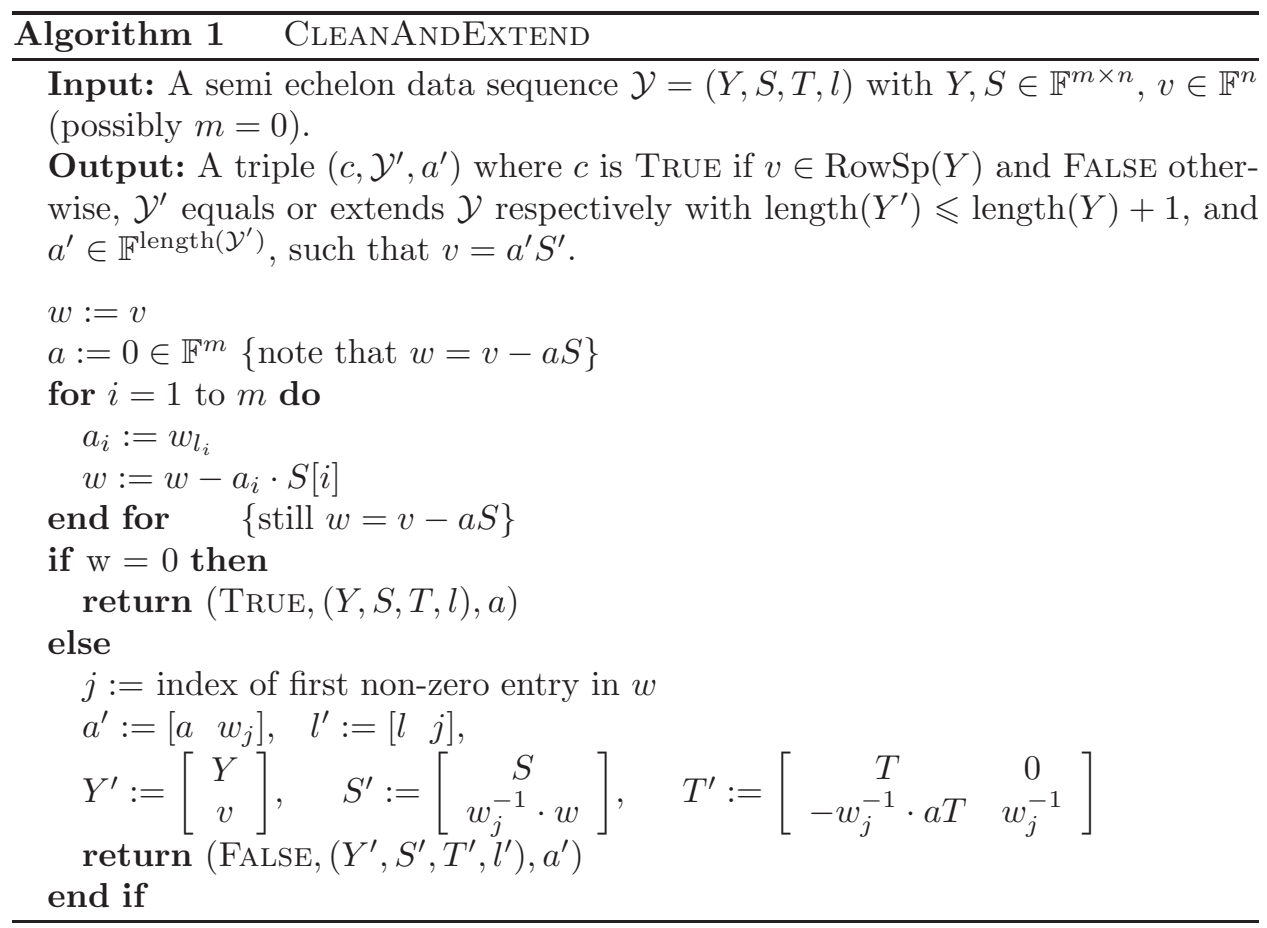


requires at most $2 m n$ field operations if $v \in \operatorname{RowSp}(Y)$, and $(2 m+1) n+(m+1)^{2}+1$ field operations otherwise.

REMARK 4.8. (a) Given a semi echelon data sequence $(Y, S, T, l)$ with $Y, S \in \mathbb{F}^{m \times n}$ and a vector $v \in \mathbb{F}^{n}$, Algorithm 1 tries to write $v$ as a linear combination of the rows of $S$. If this is not possible, it constructs an extended semi echelon data sequence.

(b) For the case of finite fields a simple and useful optimisation is to reduce, where possible, the number of operations for vectors and matrices, for example, where a vector is multiplied by the zero scalar and the result is added to some other vector. This can reduce the number of operations for sparse vectors and matrices. Our estimates for the numbers of field operations then become over-estimates.

Proof of Proposition 4.7. The proof of the correctness of Algorithm 1 is left to the reader. The for loop needs $2 m n$ field operations if we count both multiplications and additions. If $v \in \operatorname{RowSp}(Y)$ then the algorithm terminates after this loop. On the other hand, if $v \notin \operatorname{RowSp}(Y)$, then Algorithm 1 needs one inversion of the scalar $w_{j}$ plus $2 \cdot \sum_{i=1}^{m} i=m(m+1)$ field operations for the vector times matrix multiplication $a T$, because $T$ is a lower triangular matrix. This is altogether $m(m+1)+1$ operations. Finally, the scalar negation of $w_{j}^{-1}$ and the multiplication of $a T$ by $-w_{j}^{-1}$ needs another $m+1$ field operations, and a further $n$ operations are needed for the computation of $w_{j}^{-1} w$ in $S^{\prime}$. Thus the total number of field operations is at most $2 m n+(m+1)^{2}+1+n$.

Having Algorithm 1 at hand we can now present Algorithm 2, which computes relative order polynomials. Since a (non-relative) order polynomial may be regarded as a relative order polynomial with respect to the zero subspace, Algorithm 2 can also be used to compute order polynomials, starting with the trivial semi echelon data sequence, (see Remark 4.6 (b)).

Proposition 4.9 (Correctness and complexity of Alg. 2: RelativeOrdPoly).

Let $\mathcal{Y}=(Y, S, T, l)$ be a semi echelon data sequence with $Y, S \in \mathbb{F}^{m \times n}$ (possibly $m=0), v \in \mathbb{F}^{n}$, and $M \in \mathbb{F}^{n \times n}$ such that $W:=\operatorname{RowSp}(Y)$ is $M$-invariant. The output of Algorithm 2 satisfies the Output specifications, and moreover if $d>0$, then rows $m+1, \ldots, m+d$ of $Y^{\prime}$ are equal to $v, v M, \ldots, v M^{d-1}$ respectively. Algorithm 2 requires at most

$$
\begin{aligned}
2 d n^{2} & +(n+2) d+2(m+d) n+2(n+1) s^{(1)}(m+d-1, m-1)+ \\
& +s^{(2)}(m+d-1, m-1)+2 s^{(1)}(m+d, 0)
\end{aligned}
$$

elementary field operations where $s^{(1)}$ and $s^{(2)}$ are the functions defined in (1).

REMARK 4.10. Note that, if $d=0$ then $S^{\prime}=S$, so $v=b^{\prime} Y \in W$, and in this case $p=1$. Algorithm 2 successively considers the vectors $v+W, v M+W, \ldots, v M^{d}+W$ (those are the successive values of $v^{\prime}$ ) until $v M^{d}+W$ lies in the subspace of $V / W$ generated by the vectors $v+W, v M+W, \ldots, v M^{d-1}+W$. The given matrix $S$ together with Algorithm 1 defines a direct sum decomposition of the $\mathbb{F}$-vector space $V:=\mathbb{F}^{n}=W \oplus W^{\prime}$ where $W^{\prime}$ is the subspace of vectors having 0 in all positions occurring in the list $l$. Since $W^{\prime} \cong V / W$, Algorithm 2 effectively computes in $V / W$ by always 'cleaning out' vectors using $S$ first. 


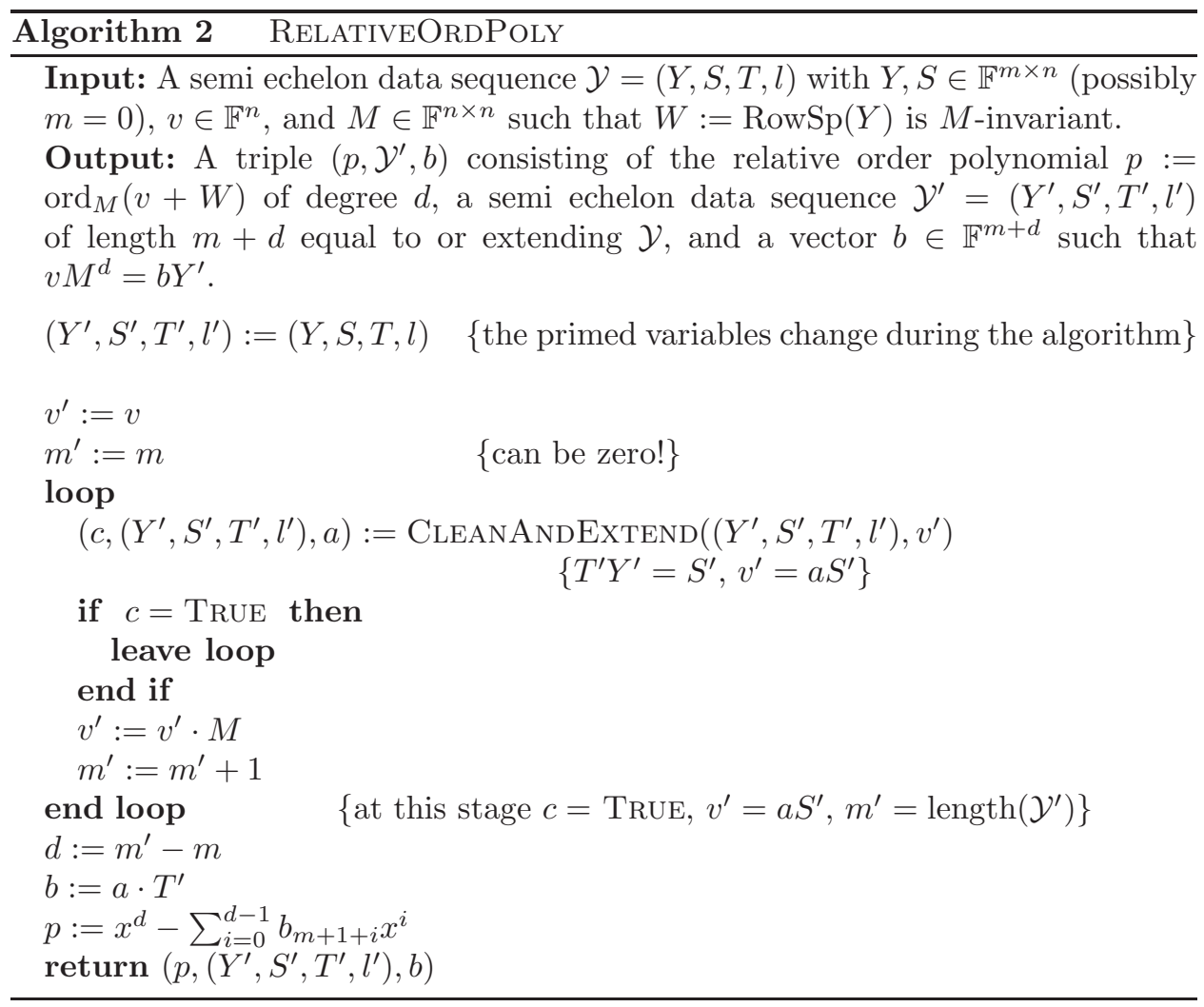

Proof of Proposition 4.9. We again leave the proof of correctness of Algorithm 2 to the reader. Algorithm 2 calls Algorithm 1 (CLEAnAndExtend) exactly $d+1$ times with the lengths of the input semi echelon data sequences being $m, m+1, \ldots, m+d$. After each but the last call to Algorithm 1 the value of $c$ returned is FALSE, and after the last call the value of $c$ is True. Thus, by Proposition 4.7, the number of steps needed for the $d+1$ runs of Algorithm 1 is at most

$$
\left(\sum_{i=m}^{m+d-1}\left((2 i+1) n+(i+1)^{2}+1\right)\right)+2(m+d) n
$$

$=s^{(2)}(m+d-1, m-1)+(2 n+2) s^{(1)}(m+d-1, m-1)+(n+2) d+2(m+d) n$.

In addition, we have to do $d$ multiplications of $v^{\prime}$ with $M$, which require $2 n^{2}$ elementary field operations each, and finally the computation of $b$ requires $2 s^{(1)}(m+d, 0)$ elementary field operations, again since $T^{\prime}$ is a lower triangular matrix. Summing up gives the expression in the statement.

We conclude this section with two lemmas that are used to compute absolute order polynomials using relative ones. We again view $V$ as an $\mathbb{F}[x]$-module by letting $x$ act like $M$. For $\left\{v^{(1)}, \ldots, v^{(m)}\right\} \subseteq V$, we denote by $\left\langle v^{(1)}, \ldots, v^{(m)}\right\rangle_{M}$ the submodule of $V$ generated by $\left\{v^{(1)}, \ldots, v^{(m)}\right\}$, that is, the smallest $M$-invariant subspace containing $\left\{v^{(1)}, \ldots, v^{(m)}\right\}$. If $m=1$ then $\left\langle v^{(1)}\right\rangle_{M}$ is the $\mathbb{F}$-span of the set $\left\{v^{(1)}, v^{(1)} M, \ldots, v^{(1)} M^{n-1}\right\}$. We call $\left\langle v^{(1)}\right\rangle_{M}$ a cyclic subspace relative to $M$. 
LEMMA 4.11 (Order polynomials in cyclic subspaces). Let $v \in V, W=\langle v\rangle_{M}<V$, and $p:=\operatorname{ord}_{M}(v)$ with $d:=\operatorname{deg}(p)$. Then for each $w \in W$, there is a unique polynomial $q \in \mathbb{F}[x]$ of degree less than $d$ such that $w=v q(M)$. Moreover,

$$
\operatorname{ord}_{M}(w)=\frac{p}{\operatorname{gcd}(p, q)}
$$

We omit the routine proof for the sake of brevity.

LEMma 4.12 (Absolute and relative order polynomials). Let $W$ be an $M$-invariant subspace of $V, v \in V$ and $q:=\operatorname{ord}_{M}(v+W) \in \mathbb{F}[x]$. Then

$$
\operatorname{ord}_{M}(v)=q \cdot \operatorname{ord}_{M}(v q(M)) .
$$

We omit the routine proof for the sake of brevity.

\section{Computing the characteristic polynomial}

In this section we present a version of a standard algorithm for computing the characteristic polynomial of a matrix together with its analysis. It differs from the standard version in its use of randomisation.

\subsection{Random vectors}

Our characteristic polynomial algorithm, and later ones, make use of the algorithms RANDOMVECTOR and RANDOMVECTOR* that produce independent uniformly distributed random vectors, and independent uniformly distributed random non-zero vectors, respectively, in a given finite vector space for which a basis is known. The algorithms are invoked for spaces $\mathbb{F}^{s}$, for $s \in \mathbb{N}$, and for subspaces of $V$ of the form

$$
V(l)=\left\{v \mid v_{l_{i}}=0 \quad \text { for } \quad 1 \leqslant i \leqslant m\right\} \quad \text { where } \quad l=\left(l_{1}, \ldots, l_{m}\right) .
$$

If $l$ is the empty sequence then $V(l)=V$. For a semi echelon data sequence $\mathcal{Y}=$ $(Y, S, T, l)$, the vector space $V$ is the sum $V=V(l) \oplus \operatorname{RowSp}(S)$.

If $b=\operatorname{RANDOMVECTOR}\left(\mathbb{F}^{\operatorname{length}(\mathcal{Y})}\right)$, then $b S$ is a uniformly distributed random vector of $\operatorname{RowSp}(S)$. Moreover we assume that for the disjoint spaces $\mathbb{F}^{\operatorname{length}(\mathcal{Y})}$ and $V(l)$ the algorithms RANDOMVECTOR and RANDOMVECTOR* are applied independently so that in particular, if $a=$ RANDOMVECTOR $^{*}(V(l))$ then the sum $a+b S$ is a uniformly distributed random vector of $V \backslash \operatorname{RowSp}(S)$.

RANDOMVECTOR and, if we neglect the possibility of obtaining the zero vector, also RANDOMVECTOR* , could proceed by selecting independent uniformly distributed random field elements as coefficients of the basis vectors. For the subspace $V(l)$, we could put zeros into the entries occurring in $l$ and make random selections of elements from $\mathbb{F}$ for each entry not in $l$. For this reason we denote by $\xi_{r}$ an upper bound for the cost of RANDOMVECTOR or RANDOMVECTOR ${ }^{*}$ applied to an $r$-dimensional space for one of these cases. If $r<s$ then $\xi_{r} \leqslant \xi_{s}$ and $\xi_{r_{1}}+\xi_{r_{2}} \leqslant \xi_{r_{1}+r_{2}}$, and we would expect $\xi_{r}$ to vary linearly with $r$. In practical implementations the cost is much less than the cost of the field operations involved in the algorithm below. 


\subsection{Characteristic polynomial algorithm}

The characteristic polynomial algorithm below would terminate successfully without making random selections of vectors. However, the use of randomisation is key to our application of this algorithm for finding minimal polynomials. As in previous sections, let $M$ be a matrix in $F^{n \times n}$ acting naturally on $V:=\mathbb{F}^{n}$.

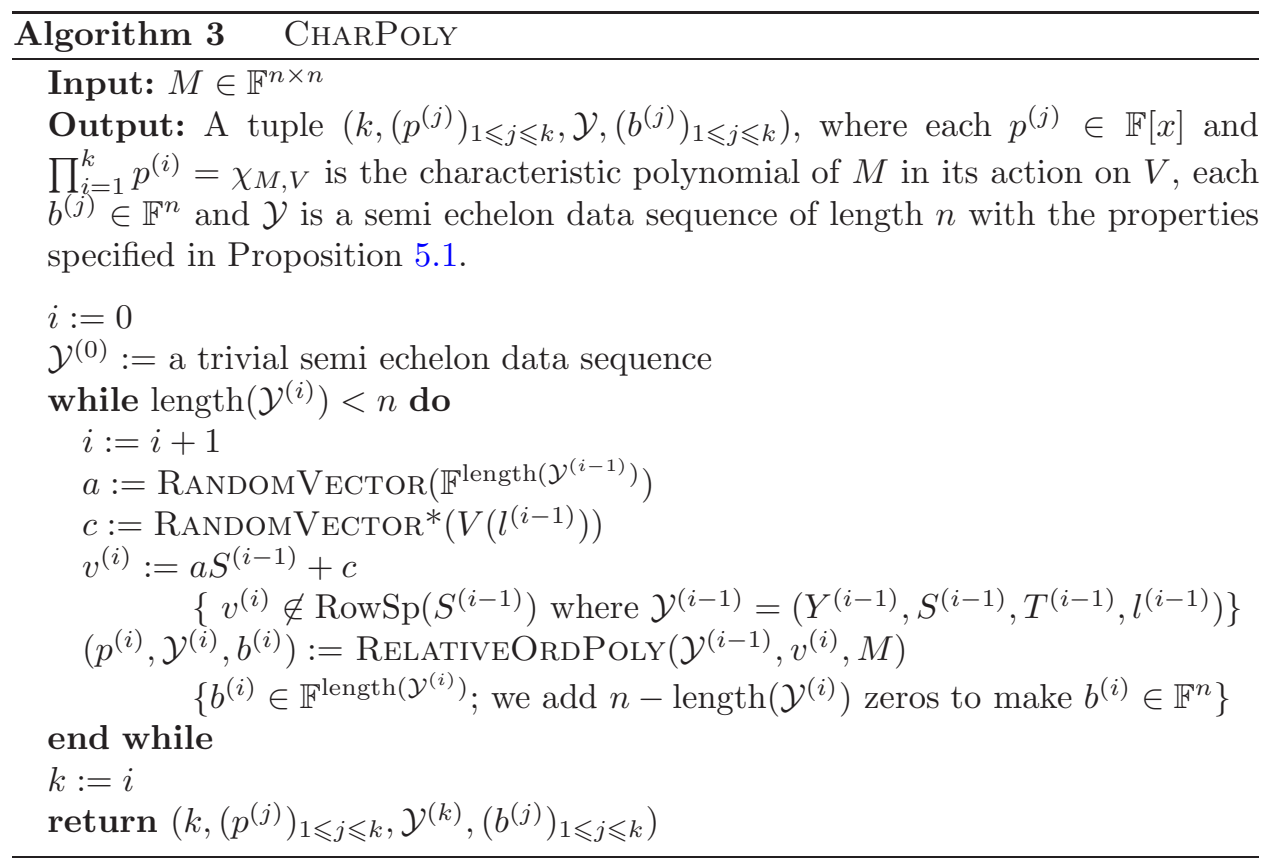

Proposition 5.1 (Correctness and complexity of Algorithm 3). Algorithm 3 satisfies the Output specifications, and furthermore $\mathcal{Y}=(Y, S, T, l)$ where $Y \in \mathbb{F}^{n \times n}$ is invertible with rows

$$
v^{(1)}, v^{(1)} M, \ldots, v^{(1)} M^{d_{1}-1}, v^{(2)}, v^{(2)} M, \ldots, v^{(2)} M^{d_{2}-1}, \ldots, v^{(k)}, v^{(k)} M, \ldots, v^{(k)} M^{d_{k}-1}
$$

where $d_{i}:=\operatorname{deg}\left(p^{(i)}\right)$ for $1 \leqslant i \leqslant k$. Further, for $1 \leqslant i \leqslant k, v^{(i)}$ is a uniformly distributed random element of $V \backslash W_{i-1}, v^{(i)} M^{d_{i}}=b^{(i)} Y$ and $p^{(i)}=\operatorname{ord}_{M}\left(v^{(i)}+\right.$ $\left.W_{i-1}\right)$, where $W_{i-1}:=\left\langle v^{(1)}, \ldots, v^{(i-1)}\right\rangle_{M}($ for $i>1)$, an $M$-invariant subspace of $V$ of dimension $s_{i-1}:=\sum_{j=1}^{i-1} d_{j}$, and $W_{0}=0$ of dimension $s_{0}=0$. Moreover, Algorithm 3 requires at most

$$
\frac{33}{6} n^{3}+4 n^{2}+\frac{3}{2} n
$$

elementary field operations, plus $k \xi_{n}$ for the $k$ calls to RANDOMVECTOR* and RANDOMVECTOR. Neglecting the latter cost this is less than $6 n^{3}$ elementary field operations, for sufficiently large $n$.

REMARK 5.2. We denote the semi echelon data sequences $\mathcal{Y}^{(i)}$ in the algorithm using indices to enable us to speak more easily about the intermediate results. However in practice we have only one variable $\mathcal{Y}=(Y, S, T, l)$, the entries of which are growing during the execution of the algorithm. 
REMARK 5.3. Note that we do not multiply together the factors of $\chi_{M, V}$ because in our application of Algorithm 3 we do not need the product itself.

Proof of Proposition 5.1. Most statements in the proposition follow immediately from Proposition 4.9: note that, because of the conventions explained in Remark $4.6(\mathrm{~b})$, in the first run of the 'while' loop $v^{(1)}=c$ is a random non-zero vector of $V$ and $p^{(1)}=\operatorname{ord}_{M}\left(v^{(1)}\right)$, and more generally, in the $i$ th run of the 'while' loop, Algorithm 3 chooses a vector $v^{(i)}$ that is a uniformly distributed random element of $V \backslash \operatorname{RowSp}\left(S^{(i-1)}\right)$ and applies Algorithm 2. This immediately establishes all statements about $(Y, S, T, l)$ including the one about the invertibility and the rows of $Y$. Also it is clear that $p^{(i)}=\operatorname{ord}_{M}\left(v^{(i)}+W_{i-1}\right)$.

Next we show that $\prod_{i=1}^{k} p^{(i)}=\chi_{M, V}$. This follows by considering the matrix $Y M Y^{-1}$, which has the same characteristic polynomial as $M$. Considering the action of $M$ with respect to the ordered basis of $\mathbb{F}^{n}$ given by the rows of $Y$, it follows from the construction that $Y M Y^{-1}$ (written with respect to the standard basis) is equal to

$$
\left[\begin{array}{cccc}
C_{1} & 0 & \cdots & 0 \\
B_{1}^{(2)} & C_{2} & \ddots & \vdots \\
\vdots & \ddots & \ddots & 0 \\
B_{1}^{(k)} & \cdots & B_{k-1}^{(k)} & C_{k}
\end{array}\right]
$$

where the matrix $C_{i}$ is the companion matrix of the polynomial $p^{(i)}$, and the $B_{j}^{(i)}$, for $2 \leqslant i \leqslant k$ and $1 \leqslant j \leqslant i-1$, are matrices in $\mathbb{F}^{d_{i} \times d_{j}}$ with one non-zero row at the bottom and all other rows zero. If $b^{(i)}=\left(b_{1}^{(i)}, \ldots, b_{n}^{(i)}\right)$, then the bottom row of $B_{j}^{(i)}$ is $\left(b_{s_{j-1}+1}^{(i)}, \ldots, b_{s_{j}}^{(i)}\right)$. With this format at hand it is clear that the characteristic polynomial of $Y M Y^{-1}$ is equal to the product $\prod_{i=1}^{k} p^{(i)}$ because the $C_{i}$ are companion matrices.

Finally we derive the statement about the number of elementary field operations needed by Algorithm 3. In the $i$ th run of the while loop, the cost of constructing the random vectors $a$ and $c$ is at most

$$
\xi_{n-\operatorname{length}\left(\mathcal{Y}^{(i-1)}\right)}+\xi_{\text {length }\left(\mathcal{Y}^{(i-1)}\right)} \leqslant \xi_{n},
$$

(see Subsection 5.1). The cost to compute $v^{(i)}$ is at most $2 s_{i-1} n$ elementary field operations, where $s_{0}=0$, and for $i \geqslant 1, s_{i}=\sum_{j=1}^{i} d_{j}$ with $d_{j}=\operatorname{deg} p^{(j)}$. The cost of applying Algorithm RelativeOrdPoly is, by Proposition 4.9, at most

$2 d_{i} n^{2}+(n+2) d_{i}+2 s_{i} n+2(n+1) s^{(1)}\left(s_{i}-1, s_{i-1}-1\right)+s^{(2)}\left(s_{i}-1, s_{i-1}-1\right)+2 s^{(1)}\left(s_{i}, 0\right)$

elementary field operations, noting that the value of ' $d$ ' is $d_{i}$, the value of ' $m$ ' is $s_{i-1}, s_{i-1}+d_{i}=s_{i}$, and $s^{(1)}, s^{(2)}$ are the functions defined in (1).

We consider the different terms one by one, summing each over $i$ from 1 to $k$. The total cost of constructing the random vectors is at most $k \xi_{n}$. Summing the terms $2 s_{i-1} n$ gives $2 n \sum_{i=1}^{k} s_{i-1}$, and summing the terms $2 d_{i} n^{2}$ gives $2 n^{3}$ since $\sum_{i=1}^{k} d_{i}=n$. Similarly, summing the terms $(n+2) d_{i}$ gives $(n+2) n$. From the terms $2 s_{i} n$ we get a contribution of $2 n \sum_{i=1}^{k} s_{i}$. The next two expressions involving the functions $s^{(1)}$ and $s^{(2)}$ sum to $2(n+1) s^{(1)}(n-1,0)=n(n+1)(n-1)$ and 
$s^{(2)}(n-1,0)=\frac{(n-1) n(2 n-1)}{6}$ respectively, using (2) and (3) and the properties noted above it. Finally, the terms $2 s^{(1)}\left(s_{i}, 0\right)$ sum to $2 \sum_{i=1}^{k} s^{(1)}\left(s_{i}, 0\right)=\sum_{i=1}^{k} s_{i}\left(s_{i}+1\right)$. Thus in total we obtain $k \xi_{n}$ plus

$$
\begin{aligned}
2 n^{3} & +n(n+1)(n-1)+\frac{(n-1) n(2 n-1)}{6}+n(n+2)+2 n \sum_{i=1}^{k} s_{i-1} \\
& +2 n \sum_{i=1}^{k} s_{i}+\sum_{i=1}^{k} s_{i}\left(s_{i}+1\right)
\end{aligned}
$$

elementary field operations. The first four of these terms sum to $\frac{10}{3} n^{3}+\frac{3}{2} n^{2}+\frac{7}{6} n$. Using Lemma 2.1,

$$
2 n \sum_{i=1}^{k} s_{i-1}+2 n \sum_{i=1}^{k} s_{i}+\sum_{i=1}^{k} s_{i}\left(s_{i}+1\right) \leqslant 2 n^{2}(n+1)+\frac{n(n+1)(n+2)}{6}
$$

so the total cost is at most

$$
\frac{33}{6} n^{3}+4 n^{2}+\frac{3}{2} n+k \xi_{n}
$$

For sufficiently large $n$ this is less than $6 n^{3}+k \xi_{n}$.

\section{Probability estimates using the structure theory for modules}

The basic idea of our minimal polynomial Algorithm 5 is to compute the order polynomials of a few random vectors under the action of a given matrix $M$ and to prove that, with high probability, their least common multiple is equal to the minimal polynomial of $M$. The purpose of this section is to use the structure theory of $V=\mathbb{F}^{n}$ as an $\mathbb{F}[x]$-module to derive probability estimates to be used in that proof.

First suppose that the characteristic polynomial of $M$ is written as a product $\chi_{M, V}=\prod_{i=1}^{t} q_{i}^{e_{i}}$ with pairwise distinct irreducible polynomials $q_{i} \in \mathbb{F}[x]$ and positive integer multiplicities $e_{i}$.

Using [7, Theorem 3.12] we can then write the $\mathbb{F}[x]$-module $V$ as a direct sum of primary cyclic modules

$$
V \cong \bigoplus_{i=1}^{t} \bigoplus_{j=1}^{m_{i}} w_{i, j} \mathbb{F}[x]
$$

such that $\operatorname{ord}_{M}\left(w_{i, j}\right)=q_{i}^{f_{i, j}}$ with $e_{i} \geqslant f_{i, 1} \geqslant f_{i, 2} \geqslant \cdots \geqslant f_{i, m_{i}} \geqslant 1$ and $\sum_{j=1}^{m_{i}} f_{i, j}=$ $e_{i}$ for $1 \leqslant i \leqslant t$.

The minimal polynomial $\mu_{M, V}$ is the least common multiple of the order polynomials of the vectors $\left(w_{i, j}\right)_{1 \leqslant i \leqslant t, 1 \leqslant j \leqslant m_{i}}$, and hence is $\mu_{M, V}=\prod_{i=1}^{t}\left(q_{i}\right)^{f_{i, 1}}$.

We use this structural description to derive the first probability bound for the case where $\mathbb{F}=\mathbb{F}_{q}$ is a finite field with $q$ elements.

Proposition 6.1 (Probability that a $q_{i}$ has equal mult. in $\mu_{M, V}$ and $\operatorname{ord}_{M}(v)$ ).

Let $\mathbb{F}=\mathbb{F}_{q}$ be a finite field with $q$ elements, let $V=\mathbb{F}^{n}$, let $U$ be a (possibly zero) $M$-invariant subspace such that the multiplicity of $q_{i}$ in $\mu_{M, U}$ is strictly smaller than in $\mu_{M, V}$, and let $v$ be a uniformly distributed random element of $V \backslash U$. Then the 
multiplicity of $q_{i}$ is the same in $\operatorname{ord}_{M}(v)$ and $\mu_{M, V}$ with probability greater than $1-q^{-\operatorname{deg} q_{i}}$.

Proof. By assumption the multiplicity of $q_{i}$ in $\mu_{M, U}$ is less that its multiplicity $f:=f_{i, 1}$ in $\mu_{M, V}$. Let $w:=w_{i, 1}$, with $w_{i, 1}$ as in (4), so that $V=X \oplus Y$ with $X, Y$ invariant under $M$ and $X=\langle w\rangle_{M}$. Then $\mu_{M, X}=q_{i}^{f}$. We may identify the primary cyclic $\mathbb{F}[x]$-module $X$ with $w \mathbb{F}[x]$, which is isomorphic to the module $\mathbb{F}[x] /\left(q_{i}^{f} \mathbb{F}[x]\right)$, and in turn this is uniserial with composition series

$$
0<\frac{q_{i}^{f-1} \mathbb{F}[x]}{q_{i}^{f} \mathbb{F}[x]}<\frac{q_{i}^{f-2} \mathbb{F}[x]}{q_{i}^{f} \mathbb{F}[x]}<\cdots<\frac{q_{i} \mathbb{F}[x]}{q_{i}^{f} \mathbb{F}[x]}<\frac{\mathbb{F}[x]}{q_{i}^{f} \mathbb{F}[x]} .
$$

Thus, $X$ has a unique maximal $\mathbb{F}[x]$-submodule, namely $X^{\prime}:=\left\langle w q_{i}(M)\right\rangle_{M}$, and $X^{\prime}$ has codimension $r:=\operatorname{deg}\left(q_{i}\right)$ in $X$.

As discussed above, each vector $v \in V$ has a unique expression as $v=x+y$ with $x \in X, y \in Y$. Moreover $\operatorname{ord}_{M}(v)$ is the least common multiple of $\operatorname{ord}_{M}(x)$ and $\operatorname{ord}_{M}(y)$. In particular, if $x \notin X^{\prime}$, then $\operatorname{ord}_{M}(x)=q_{i}^{f}$ and hence the multiplicity of $q_{i}$ in $\operatorname{ord}_{M}(v)$ and $\mu_{M, V}$ is the same. The number of vectors $v=x+y$ with $x \notin X^{\prime}$ is

$$
\left|X \backslash X^{\prime}\right| \cdot|Y|=\left(1-\frac{1}{q^{r}}\right)|X| \cdot|Y|=\left(1-\frac{1}{q^{r}}\right) q^{n} .
$$

Each of these vectors $v$ lies in $V \backslash U$ since the multiplicity of $q_{i}$ in $\mu_{M, U}$ is less than $f$. Thus the probability, for a uniformly distributed random $v \in V \backslash U$, that the multiplicity of $q_{i}$ in $\operatorname{ord}_{M}(v)$ and $\mu_{M, V}$ is the same is at least

$$
\left(1-\frac{1}{q^{r}}\right) \frac{q^{n}}{|V \backslash U|}>1-\frac{1}{q^{r}}
$$

REMARK 6.2. If for some irreducible factor $q_{i}$ we have $m_{i}>1$ and $f_{i, 1}=f_{i, 2}$, then the above probability is even higher, because we can apply the above argument independently to two or more summands $w_{i, 1} \mathbb{F}[x]$ and $w_{i, 2} \mathbb{F}[x]$.

We now give a second probability bound which will be crucial in our Monte Carlo algorithm to compute the minimal polynomial. In that algorithm we choose a sequence of vectors $v^{(1)}, \ldots v^{(u)}$ such that $v^{(1)}$ is a uniformly distributed random element of $V \backslash\{0\}$, and for $i \geqslant 2$ we choose $v^{(i)}$ as a uniformly distributed random element of $V \backslash U$, where $U=\left\langle v^{(1)}, \ldots, v^{(i-1)}\right\rangle_{M}$. We hope to find $\mu_{M, V}$ as the least common multiple of the orders of these vectors.

Proposition 6.3 (Probability that an lcm of order polynomials equals $\mu_{M, V}$ ).

Let $\mathbb{F}=\mathbb{F}_{q}$ be a finite field with $q$ elements. Suppose a sequence of vectors $v^{(1)}, \ldots, v^{(u)} \in V$ is chosen as follows: $v^{(1)}$ is a uniformly distributed random element of $V \backslash\{0\}$, and for $i>1, v^{(i)}$ is a uniformly distributed random element of $V \backslash\left\langle v^{(1)}, \ldots, v^{(i-1)}\right\rangle_{M}$. Let

$$
f:=\operatorname{lcm}\left(\operatorname{ord}_{M}\left(v^{(1)}\right), \operatorname{ord}_{M}\left(v^{(2)}\right), \ldots, \operatorname{ord}_{M}\left(v^{(u)}\right)\right) .
$$

Then the probability that $f=\mu_{M, V}$ is greater than

$$
1-\sum_{i=1}^{t} q^{-u \operatorname{deg} q_{i}}
$$


Proof. Consider the random experiment described in the statement. We first examine one irreducible factor $q_{i}$. Let $E_{i}$ denote the event that the multiplicity of $q_{i}$ in $f$ is strictly smaller than the multiplicity $f_{i, 1}$ of $q_{i}$ in $\mu_{M, V}$. Furthermore, for $1 \leqslant j \leqslant u$, let $F_{j}$ be the event that the multiplicity of $q_{i}$ in $\operatorname{ord}_{M}\left(v^{(j)}\right)$ is strictly smaller than $f_{i, 1}$.

Note that the $F_{j}$ are not stochastically independent since we choose $v^{(j)}$ outside of $\left\langle v^{(1)}, \ldots, v^{(j-1)}\right\rangle_{M}$. However, $E_{i}=F_{1} \cap F_{2} \cap \cdots \cap F_{u}$ because $f$ is the least common multiple of the order polynomials of the $v^{(j)}$. By Proposition 6.1 applied with $U=\{0\}$, the probability $\operatorname{Prob}\left(F_{1}\right)$ is less than $q^{-\operatorname{deg} q_{i}}$. Moreover, in the situation that $F_{1} \cap \cdots \cap F_{j}$ holds and $j<u$, we apply Proposition 6.1 with the subspace $U:=\left\langle v^{(1)}, \ldots, v^{(j)}\right\rangle_{M}$ to conclude that the conditional probability $\operatorname{Prob}\left(F_{j+1} \mid F_{1} \cap\right.$ $\left.\cdots \cap F_{j}\right)$ is less than $q^{-\operatorname{deg} q_{i}}$. Thus we have

$$
\begin{aligned}
\operatorname{Prob}\left(E_{i}\right)=\operatorname{Prob}\left(F_{1}\right) & \cdot \operatorname{Prob}\left(F_{2} \mid F_{1}\right) \cdot \operatorname{Prob}\left(F_{3} \mid F_{1} \cap F_{2}\right) \cdots \\
& \ldots \operatorname{Prob}\left(F_{u} \mid F_{1} \cap \cdots \cap F_{u-1}\right)<q^{-u \operatorname{deg} q_{i}} .
\end{aligned}
$$

Finally we consider all the different irreducible factors $q_{i}$.

Even though the events $E_{1}, \ldots, E_{t}$ may not be stochastically independent, we have

$$
\operatorname{Prob}\left(E_{1} \cup \cdots \cup E_{t}\right) \leqslant \sum_{i=1}^{t} \operatorname{Prob}\left(E_{i}\right)<\sum_{i=1}^{t} q^{-u \operatorname{deg} q_{i}}
$$

as claimed.

\section{Computing minimal polynomials}

Our minimal polynomial algorithm runs Algorithm 3 as its first step. So assume, from now on, that we have already run Algorithm 3 and obtained all the output it produces, in particular the basis given by the rows of the matrix $Y$ (as in Proposition 5.1),

$$
\left(v^{(1)}, v^{(1)} M, \ldots, v^{(1)} M^{d_{1}-1}, \ldots, v^{(k)}, v^{(k)} M, \ldots, v^{(k)} M^{d_{k}-1}\right)
$$

the relative order polynomials $p^{(i)}=\operatorname{ord}_{M}\left(v^{(i)}+W_{i-1}\right)$, and the vectors $b^{(i)}$ for $1 \leqslant i \leqslant k$. Also assume that we have factorised all the polynomials $p^{(i)}$ as products $p^{(i)}=\prod_{j=1}^{t} q_{j}^{e_{i, j}}$ of irreducible polynomials $\left(q_{j}\right)_{1 \leqslant j \leqslant t}$.

The matrices $M$ and $Y M Y^{-1}$ have the same characteristic and minimal polynomials. Also the order polynomials $\operatorname{ord}_{M}(v)$ and $\operatorname{ord}_{Y M Y^{-1}}\left(v Y^{-1}\right)$ are equal for every $v \in V$ and thus also the order polynomials $\operatorname{ord}_{M}(v Y)$ and $\operatorname{ord}_{Y M Y^{-1}}(v)$ are equal for every $v \in V$.

For the convenience of the reader we display the matrix $Y M Y^{-1}$ in Figure 1. Note in particular that the matrix is sparse, provided that the degrees $d_{i}$ are not too small. Due to the special form of $Y M Y^{-1}$ it is much more efficient to compute the images of vectors under $Y M Y^{-1}$, than under $M$. This is crucial in the analysis of our algorithms. Therefore we will from now on do all computations of order polynomials with respect to $Y M Y^{-1}$.

Set $M^{\prime}:=Y M Y^{-1}$ and $W_{i}^{\prime}:=W_{i} Y^{-1}$ for $1 \leqslant i \leqslant k$. Note that we have $v^{(i)}=$ $e^{\left(s_{i-1}+1\right)} Y$ for $1 \leqslant i \leqslant k$ where $e^{(1)}, \ldots, e^{(n)}$ is the standard basis of $\mathbb{F}^{n}$. (That is, $e^{(i)}$ contains exactly one 1 in position $i$ and otherwise zeros. Recall that $s_{i}=\sum_{j=1}^{i} d_{j}$ 
Figure 1: Overview of the matrix $Y M Y^{-1}$

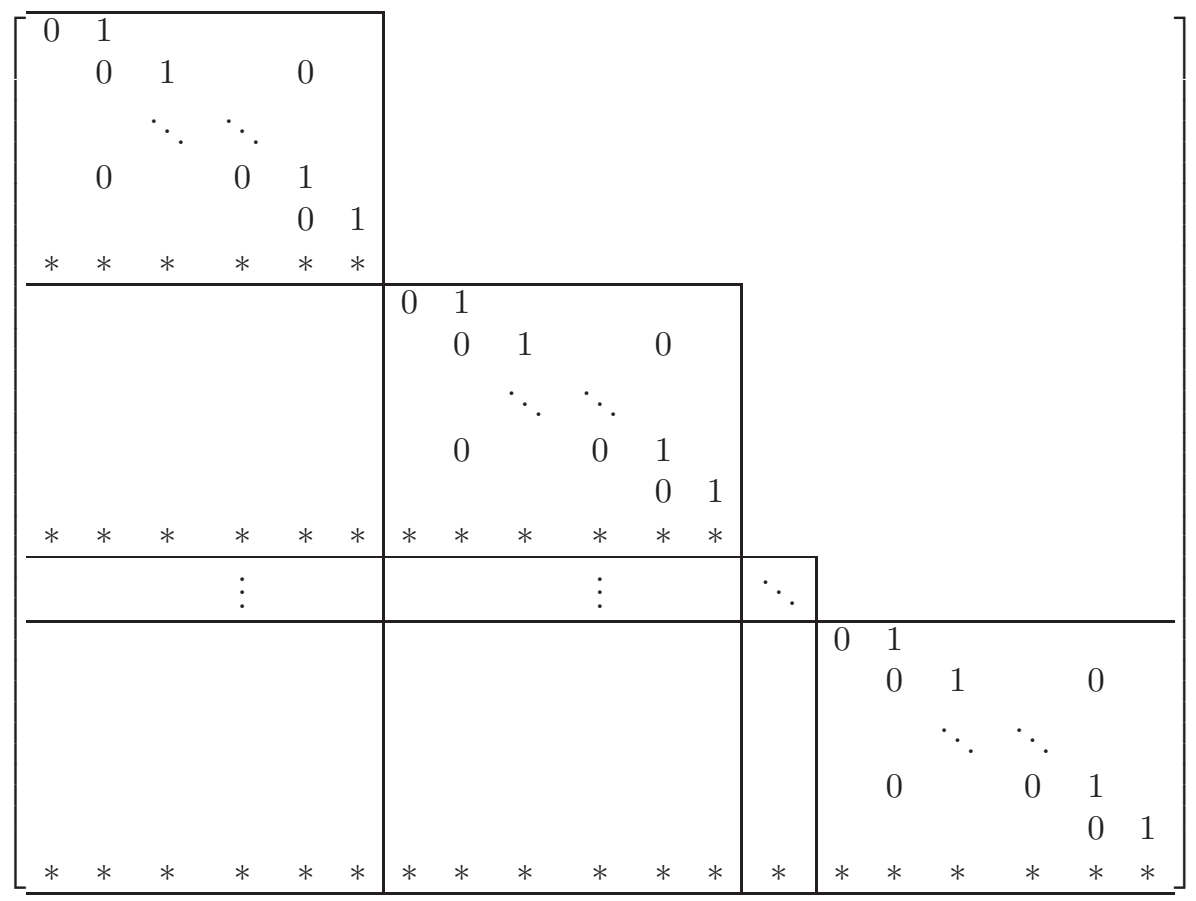

with $s_{0}=0$.) Furthermore, for $1 \leqslant i \leqslant k$, the space $W_{i}=\left\langle v^{(1)}, \ldots, v^{(i)}\right\rangle_{M}$ is equal to the space $\left\{v Y \mid v \in \mathbb{F}^{n}\right.$ with $v_{j}=0$ for $\left.j>s_{i}\right\}$. Thus, the space $W_{i}^{\prime}$ is the $\mathbb{F}$-linear span $\left\langle e^{(1)}, e^{(2)}, \ldots, e^{\left(s_{i}\right)}\right\rangle_{\mathbb{F}}$ and we have a filtration

$$
0=W_{0}^{\prime}<W_{1}^{\prime}<W_{2}^{\prime}<\cdots<W_{k}^{\prime}=V
$$

such that each quotient $W_{i}^{\prime} / W_{i-1}^{\prime}$ is an $M^{\prime}$-cyclic space generated by the coset represented by the standard basis vector $e^{\left(s_{i-1}+1\right)}$.

We begin by presenting Algorithm 4 which computes the absolute order polynomial of a vector with respect to the matrix $Y M Y^{-1}$, using all the data acquired during Algorithm 3. We will apply this later in the minimal polynomial algorithm to the first few of the vectors $e^{\left(s_{i-1}+1\right)}$ produced during a run of Algorithm 3. Note that for the analysis it is crucial that a number $z$ such that the vector $v$ lies in $W_{z}^{\prime}$ is given as input to the algorithm.

Proposition 7.1 (Correctness and complexity of Algorithm 4: OrdPoly).

Let $\mathbb{F}=\mathbb{F}_{q}$ be a field with $q$ elements. The output of Algorithm 4 satisfies the Output specifications. Moreover, Algorithm 4 requires at most

$$
\sum_{j=1}^{z}\left(4 d_{j}^{2}+3 d_{j} s_{j}+2 d_{j} \sum_{r=1}^{j} s_{r}\right) \leqslant\left(\frac{z}{2}+9\right) s_{z}^{2}
$$

elementary field operations, where $d_{j}=\operatorname{deg} p^{(j)}, s_{j}=\sum_{r=1}^{j} d_{j}$ for $j \geqslant 1$ and $s_{0}=0$; and this is less than $n^{3}$ for $n$ sufficiently large. 


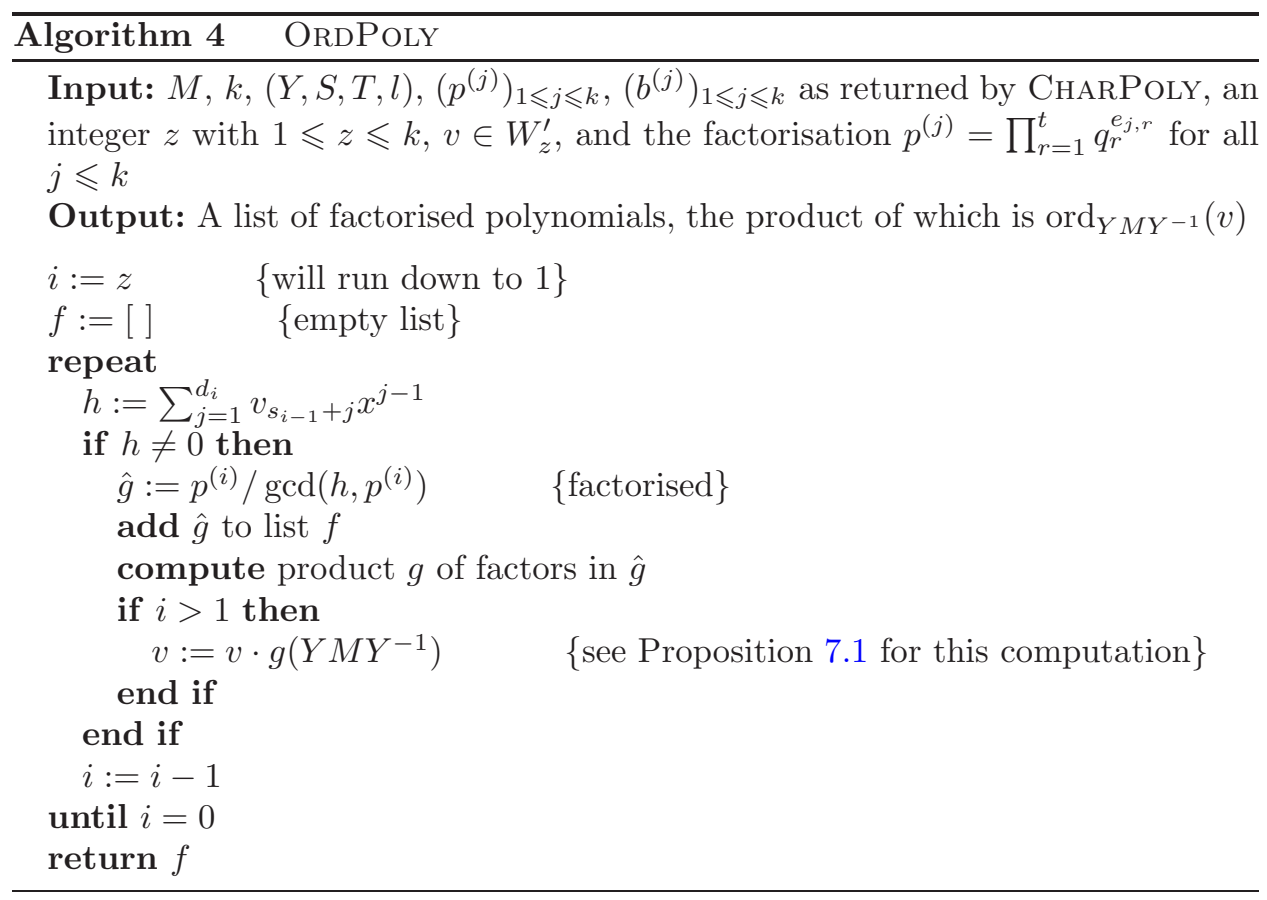

Proof. Since we are computing an order polynomial with respect to the matrix $M^{\prime}=Y M Y^{-1}$ we can always use the form of this matrix as displayed in Figure 1.

The basic idea of Algorithm 4 is to use Lemmas 4.11 and 4.12 applied to the filtration

$$
0=W_{0}^{\prime}<W_{1}^{\prime}<W_{2}^{\prime}<\cdots<W_{k}^{\prime}=V
$$

Starting with $i:=z$ and the original $v$ lying in the space $W_{z}^{\prime}$, the variable $i$ runs downwards until 1. In each step, Algorithm 4 computes the relative order polynomial $g:=\operatorname{ord}_{M^{\prime}}\left(v+W_{i-1}^{\prime}\right)$ for the then current value of $v \in W_{i}^{\prime}$. This assertion follows from Lemma 4.11 noting that, by our discussion above, $p^{(i)}=$ $\operatorname{ord}_{M}\left(v^{(i)}+W_{i}\right)=\operatorname{ord}_{M^{\prime}}\left(e^{\left(s_{i-1}+1\right)}+W_{i-1}^{\prime}\right)$. Next $v g\left(M^{\prime}\right)$ is evaluated, which lies in $W_{i-1}^{\prime}$ by Lemma 4.3 , and the induction can go on with $i$ replaced by $i-1$. The product of the polynomials in the list $f$ returned is the product of all the relative order polynomials computed in the repeat loop, and this is equal to $\operatorname{ord}_{M^{\prime}}(v)$, by Lemma 4.12.

To count the number of elementary field operations is a bit complicated here. Note first that by assumption we already know a factorisation of all the $p^{(i)}=$ $\prod_{j=1}^{t} q_{j}^{e_{i, j}}$ into irreducible factors. Now $\operatorname{gcd}\left(h, p^{(i)}\right)$ is equal to the product of the greatest common divisors $\operatorname{gcd}\left(h, q_{j}^{e_{i, j}}\right)$, for $j \leqslant t$. Since the degrees of the polynomials $q_{j}^{e_{i, j}}$ sum up to the degree $d_{i}$ of $p^{(i)}$, finding these gcd's, by Proposition 3.1, requires at most

$$
2(\operatorname{deg}(h)+1) \cdot \sum_{j=1}^{t}\left(\operatorname{deg}\left(q_{j}\right) e_{i, j}+1\right)
$$


field operations, which is at most $4 d_{i}^{2}$ since $\operatorname{deg}\left(q_{j}\right) e_{i, j}+1 \leqslant 2 \operatorname{deg}\left(q_{j}\right) e_{i, j}$. Note that this is a rather crude estimate. At this stage we know all multiplicities of the $q_{j}$ in $\operatorname{gcd}\left(h, p^{(i)}\right)$ and thus in $g:=p^{(i)} / \operatorname{gcd}\left(h, p^{(i)}\right)$. Thus we have computed $g$ in factorised form, which is denoted by $\hat{g}$ in Algorithm 4.

Now we discuss the number of operations needed to evaluate $v g\left(M^{\prime}\right)$. Due to the sparseness of $M^{\prime}$, a multiplication of a vector $w$ of $W_{i}^{\prime}$ from the right by $M^{\prime}$ needs only a shift (which we neglect here) and an addition of a multiple of the non-zero part of $b^{(r)}$ for $1 \leqslant r \leqslant i$ requiring $2 s_{r}$ operations for each $r$. Thus computing $w M^{\prime}$ requires at most $\sum_{r=1}^{i} 2 s_{r}$ elementary operations. Note that $w M^{\prime}$ lies in $W_{i}^{\prime}$ still. If $f(x) \in \mathbb{F}_{q}[x]$ of degree $d$, say $f(x)=\sum_{r=0}^{d} c_{r} x^{r}$, then we can compute $w f\left(M^{\prime}\right)=\sum_{r=0}^{d} c_{r} w\left(M^{\prime}\right)^{r}$ by first computing $w\left(M^{\prime}\right)^{r} \in W_{i}^{\prime}$ for $1 \leqslant r \leqslant d$ at a cost of at most $2 d \sum_{r=1}^{i} s_{r}$, next computing $c_{r} w\left(M^{\prime}\right)^{r}$ for $0 \leqslant r \leqslant d$ at a cost of at most $(d+1) s_{i}$, and then adding these vectors at a further cost of at most $d s_{i}$, making a total cost to compute $w f\left(M^{\prime}\right)$ of at most $(2 d+1) s_{i}+2 d \sum_{r=1}^{i} s_{r}$ elementary field operations.

The polynomial $g(x)$ is available in factorised form, say $g(x)=\prod_{s=1}^{u} f_{s}(x)$ with $\operatorname{deg} f_{s}=m_{s}$, where $\sum_{s=1}^{u} m_{s}=\operatorname{deg} g=d_{i}$. From the previous paragraph we see that $v g\left(M^{\prime}\right)$ can be computed at a cost of at most

$$
\sum_{s=1}^{u}\left(\left(2 m_{s}+1\right) s_{i}+2 m_{s} \sum_{r=1}^{i} s_{r}\right)=\left(2 d_{i}+u\right) s_{i}+2 d_{i} \sum_{r=1}^{i} s_{r} \leqslant 3 d_{i} s_{i}+2 d_{i} \sum_{r=1}^{i} s_{r} .
$$

Subsequent runs of the repeat loop require similar numbers of elementary operations, with $i$ replaced by $j$ where $i-1 \geqslant j \geqslant 1$. Thus Algorithm 4 needs at most

$$
\sum_{j=1}^{z}\left(4 d_{j}^{2}+3 d_{j} s_{j}+2 d_{j} \sum_{r=1}^{j} s_{r}\right)
$$

elementary field operations, as claimed in the proposition.

To find a simpler upper bound we look at the terms one by one. The last and most important term can be bounded by

$$
\begin{aligned}
2 \sum_{j=1}^{z}\left(d_{j} \sum_{r=1}^{j} s_{r}\right) & =2 \sum_{r=1}^{z} s_{r}\left(\sum_{j=r}^{z} d_{j}\right)=2 \sum_{r=1}^{z} s_{r}\left(s_{z}-s_{r-1}\right) \\
& =2 \sum_{r=1}^{z} s_{r}\left(s_{z}-s_{r}\right)+2 \sum_{r=1}^{z} s_{r} d_{r} \leqslant\left(\frac{z}{2}+2\right) \cdot s_{z}^{2}
\end{aligned}
$$

since the function $t\left(s_{z}-t\right)$ has maximum value $s_{z}^{2} / 4$ for $t$ in the interval $\left[0, s_{z}\right]$.

The second term $3 \sum_{j=1}^{z} d_{j} s_{j}$ is at most $3 s_{z}^{2}$, and the term $4 \sum_{j=1}^{z} d_{j}^{2}$ is at most $4 s_{z} \sum_{j=1}^{z} d_{j}=4 s_{z}^{2}$.

Altogether this amounts to a bound of $\left(\frac{z}{2}+9\right) s_{z}^{2}$ as claimed. Asymptotically, this is bounded above by $n^{3}$ in the worst case as $n \rightarrow \infty$.

Now we present our main procedure, Algorithm 5. 


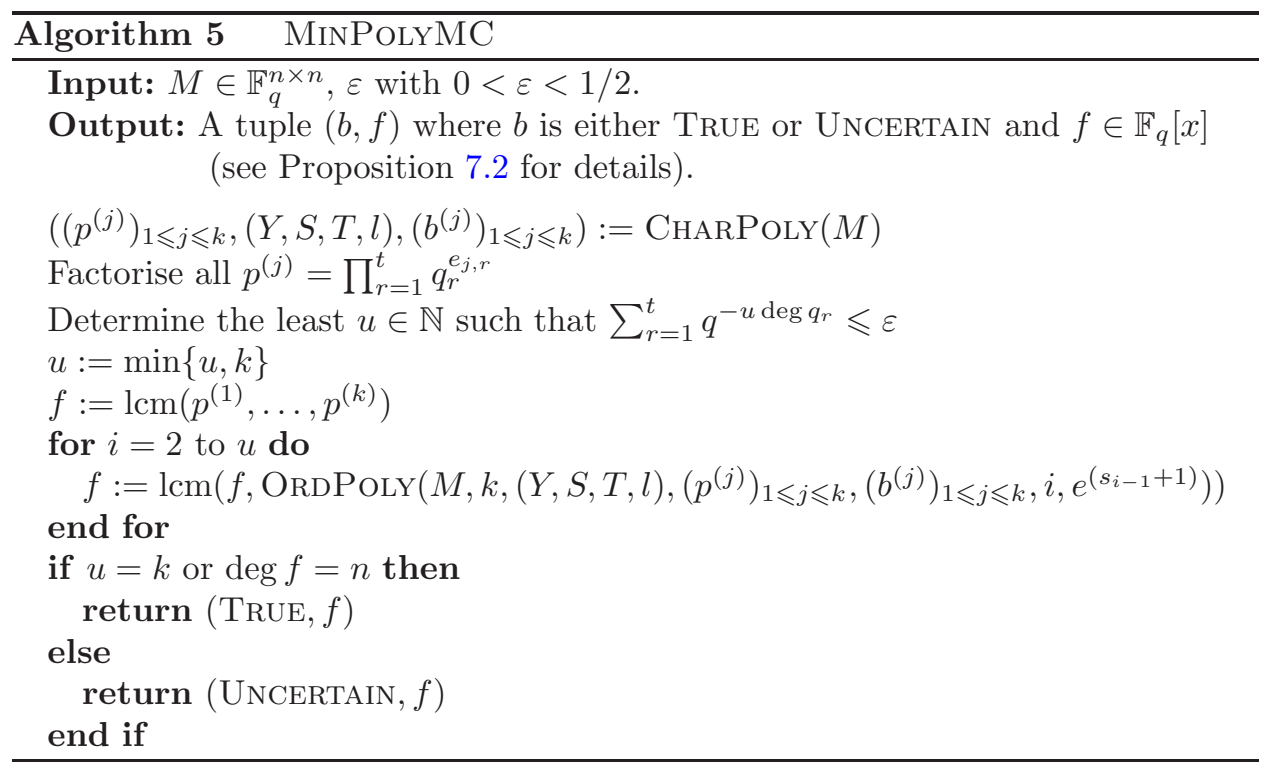

Proposition 7.2 (Correctness and complexity of Algorithm 5: MinPolyMC).

Given a matrix $M \in \mathbb{F}_{q}^{n \times n}$ and a number $\varepsilon$ with $0<\varepsilon<1 / 2$, Algorithm 5 returns a tuple $(b, f)$, where $b$ is either TRUE or UNCERTAIN and $f \in \mathbb{F}_{q}[x]$ is a polynomial. With probability at least $1-\varepsilon$ the polynomial $f=\mu_{M, \mathbb{F}_{q}^{n}}$, and if $b=$ TRUE then $f=\mu_{M, \mathbb{F}_{q}^{n}}$ is guaranteed. Moreover, if $f \neq \mu_{M, \mathbb{F}_{q}^{n}}$, then $f$ is a proper divisor of $\mu_{M, \mathbb{F}_{q}^{n}}$ and every irreducible factor of $\mu_{M, \mathbb{F}_{q}^{n}}$ divides $f$.

The number of elementary field operations needed by Algorithm 5 is bounded above by

$$
\operatorname{char}(n, q)+\operatorname{fact}(n, q)+\sum_{i=1}^{u}\left(\frac{i}{2}+9\right) s_{i}^{2}
$$

where $\operatorname{char}(n, q)$ is an upper bound for the number of elementary field operations needed to compute the characteristic polynomial (see Proposition 5.1), fact $(n, q)$ is an upper bound for the number of elementary field operations needed to factorise each of a set of polynomials over $\mathbb{F}_{q}$ whose degrees sum to $n$ (see Subsection 3.1). Moreover either $u=k$, or $u<k$ and $\sum_{j=1}^{t} q^{-u \operatorname{deg} q_{j}} \leqslant \varepsilon$.

For $n$ sufficiently large and fixed $\varepsilon$, this is less than

$$
6 n^{3}+\operatorname{fact}(n, q)+\frac{1}{3}\left\lceil\frac{\log n-\log \varepsilon}{\log q}\right\rceil^{2} \cdot n^{2}
$$

which is less than $7 n^{3}+$ fact $(n, q)$ (plus the cost of computing at most $n$ random vectors in Algorithm 3).

REMARK 7.3. Note that if we use a randomised polynomial factorisation algorithm (necessary for large $q$ ), then the algorithm can be modified to allow for a possible failure of factorisation of the 'Factorise' step (line 2). Thus Theorem 1.1 follows from Proposition 5. An upper bound for the term fact $(n, q)$ in the complexity bound is given in Remark 3.3, and this yields an upper bound in Proposition 7.2 of $O\left(n^{3} \log ^{3} q\right)$ for $n$ sufficiently large and fixed $\varepsilon$. 
REMARK 7.4. Algorithm 5 can be changed into a deterministic one by running the ' $i$-loop' for $i$ up to $k$, instead of $u$. An upper bound of the cost is then given by replacing $u$ by $k$ in the formula for the cost in Proposition 7.2.

If $k>u$, then these additional $k-u$ runs of the ' $i$-loop' may be viewed as a 'verification algorithm'. By Proposition 5, the additional cost of these extra runs is

$$
\sum_{i=u+1}^{k}\left(\frac{i}{2}+9\right) s_{i}^{2} \leqslant s_{k}^{2}\left(\frac{(k+u+1)(k-u)}{4}+9(k-u)\right)=s_{k}^{2} \frac{(k-u)(k+u+37)}{4}
$$

and for sufficiently large $n$ this cost is less than $n^{4} / 4$ field operations.

Proof of Proposition 7.2. Algorithm 5 first computes the characteristic polynomial of $M$ in its action on $\mathbb{F}_{q}^{n}$ and its factorisation. This computation provides firstly the irreducible factors $q_{j}$ of the minimal polynomial that allow us to determine $u$, and secondly the input needed for running Algorithm 4 to compute the order polynomials of $v^{(2)}, \ldots, v^{(u)}$. Thirdly, it also yields a nice base change matrix $Y$ such that these order polynomials with respect to the matrix $M$ can in fact be determined using Algorithm 2 for the vectors $e^{\left(s_{1}+1\right)}, \ldots, e^{\left(s_{u-1}+1\right)}$ since we have $\operatorname{ord}_{M}\left(v^{(i)}\right)=$ $\operatorname{ord}_{Y M Y^{-1}}\left(e^{\left(s_{i-1}+1\right.}\right)$ for $1 \leqslant i \leqslant k$. Note that $p^{(1)}=\operatorname{ord}_{Y M Y^{-1}}\left(e^{(1)}\right)=\operatorname{ord}_{M}\left(v^{(1)}\right)$. By Proposition 5.1, the vector $v^{(j)}$ is a uniformly distributed random element of $V \backslash\{0\}$ if $j=1$, or $V \backslash\left\langle v^{(1)}, \ldots, v^{(j-1)}\right\rangle_{M}$ if $j>1$. Hence, by Propositions 6.3 and 7.1, the probability that $f$ after termination of Algorithm 5 is equal to $\mu_{M, \mathbb{F}_{q}^{n}}$ is at least $1-\varepsilon$.

From the discussion at the beginning of Section $6, \mu_{M, \mathbb{F}_{q}^{n}}$ is the least common multiple of the $k$ polynomials $\operatorname{ord}_{M}\left(v^{(1)}\right), \ldots, \operatorname{ord}_{M}\left(v^{(k)}\right)$, and hence if $u=k$ then $f=\mu_{M, \mathbb{F}_{q}^{n}}$. This also implies, since the initial value of $f$ is $\operatorname{lcm}\left(p^{(1)}, \ldots, p^{(k)}\right)$, that the returned polynomial $f$ divides $\mu_{M, \mathbb{F}_{q}^{n}}$ and every irreducible factor of $\mu_{M, \mathbb{F}_{q}^{n}}$ divides $f$. In particular, if $\operatorname{deg} f=n$ then we must have $f=\chi_{M, \mathbb{F}_{q}^{n}}=\mu_{M, \mathbb{F}_{q}^{n}}$. Thus if (TRUE, $f$ ) is returned then $f=\mu_{M, \mathbb{F}_{q}^{n}}$ is guaranteed.

The number of elementary field operations needed follows from Propositions 5.1 and 7.1 and summing. Note that, after the factorisations computed in line 2 of the algorithm, we neglect the forming of least common multiples and the products here, because all results from Algorithm 4 come already factorised into irreducible factors. We can thus compute the least common multiples by taking maximums of multiplicities. Hence the first displayed upper bound is proved.

For the asymptotic complexity bound we have to consider the initial value of the number $u$, namely the least integer $u$ such that $\sum_{j=1}^{t} q^{-u \operatorname{deg} q_{j}} \leqslant \varepsilon$. The largest value of this sum occurs when all the $q_{j}$ have degree 1 , and as there are then at most $n$ such polynomials, $\sum_{j=1}^{t} q^{-u \operatorname{deg} q_{j}} \leqslant n q^{-u}$. Thus $u$ is at most the least integer such that $n q^{-u} \leqslant \varepsilon$, namely

$$
u_{0}:=\left\lceil\frac{\log n+\log \left(\varepsilon^{-1}\right)}{\log q}\right\rceil
$$

and the value of $u$ used in the algorithm is at most $\min \left\{u_{0}, k\right\} \leqslant u_{0}$. By Proposition 5.1, the asymptotic value of $\operatorname{char}(n, q)$ is less than $6 n^{3}$ for $n$ sufficiently large, (plus the cost $k \xi_{n}$ of making $k$ random selections of vectors). By Proposition 7.1, the number of elementary field operations used for the computation of the $u \leqslant u_{0}$ 
order polynomials is at most

$$
\sum_{i=1}^{u}\left(\frac{i}{2}+9\right) s_{i}^{2} \leqslant \frac{u(u+1)}{4} s_{u}^{2}+9 u s_{u}^{2} \leqslant u_{0}\left(\frac{u_{0}+37}{4}\right) n^{2} .
$$

which, for sufficiently large $n$ and fixed $\epsilon$, is less than $\frac{1}{3} u_{0}^{2} n^{2}<n^{3}$.

\section{Deterministic verification}

In this section we explain how the probabilistic result of our Monte Carlo algorithm can be verified deterministically. We begin by discussing cases that can be handled rather cheaply, before we present several general verification procedures, all of which, unfortunately, have a worst-case cost of $O\left(n^{4}\right)$ field operations.

All notation from previous sections remains in force. The first result follows immediately from Proposition 7.2.

Proposition 8.1 (Cases, in which the result is already proven to be correct). If the output polynomial of Algorithm 5 is $\chi_{M, \mathbb{F}_{q}^{n}}$, then the output is (TRUE, $\chi_{M, \mathbb{F}_{q}^{n}}$ ) and is correct.

For the next result observe that, if Algorithm 5 is modified so that the for loop is run $k$ times, then the resulting polynomial $f$ is guaranteed to be the minimal polynomial, giving a deterministic algorithm with proven result. (Proof of correctness is given in the proof of Proposition 7.2.)

Proposition 8.2 (Case of few random vectors chosen during comp. of $\chi_{M, \mathbb{F}_{q}^{n}}$ ).

If $k \leqslant \sqrt{n}$, and the for loop in Algorithm 5 is run $k$ times, then the output

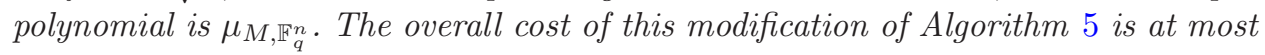

$$
\operatorname{char}(n, q)+\operatorname{fact}(n, q)+\frac{1}{4} n^{3}+\frac{37}{4} n^{5 / 2}
$$

elementary field operations.

Proof. The only change to the complexity estimate is for the number of elementary field operations in the second last line of the proof of Proposition 7.2:

$$
\sum_{i=1}^{k}\left(\frac{i}{2}+9\right) s_{i}^{2} \leqslant \frac{k(k+1)}{4} s_{k}^{2}+9 k s_{k}^{2} \leqslant \frac{k(k+1)}{4} n^{2}+9 k n^{2} \leqslant \frac{1}{4} n^{3}+\frac{37}{4} n^{5 / 2} .
$$

The rest follows from Proposition 7.2.

For the case of large $k$, we may use the procedure suggested in Remark7.4 as a verification algorithm, at a cost of $O\left(k^{2} n^{2}\right)$ field operations. Two alternative verification procedures are given below. The first involving evaluation on vectors is given in Proposition 8.3, and the second using null space computations is given in Proposition 8.7.

Proposition 8.3 (Verification by evaluation on vectors).

For the output (UNCERTAIN, $f$ ) of Algorithm 5 one can verify $f=\mu_{M, \mathbb{F}_{q}^{n}}$ using at most $d n(k-u)(k+u+4)$ elementary field operations where $u$ and $k$ are as in Proposition 7.2 and $d=\operatorname{deg} f$. 
Proof. The idea here is to check whether $e^{\left(s_{i-1}+1\right)} f\left(Y M Y^{-1}\right)$ is equal to zero, for $u+1 \leqslant i \leqslant k$, by direct evaluation using the techniques described in the proof of Propostion 7.1. Recall first that the result $f$ comes in factorised form. Since $e^{\left(s_{i-1}+1\right)}$ lies in $W_{i}^{\prime}$ the arguments in the proof of Proposition 7.1 show that this evaluation can be done using at most $3 d s_{i}+2 d \sum_{r=1}^{i} s_{r}$ elementary field operations. Thus, an upper bound for the total cost for all these evaluations is

$$
3 d \sum_{i=u+1}^{k} s_{i}+2 d \sum_{i=u+1}^{k} \sum_{r=1}^{i} s_{r} .
$$

The first term is bounded above by $3 d n(k-u)$. As to the second term, for $1 \leqslant j \leqslant$ $u+1$, the value $s_{j}$ occurs in this expression with coefficient $2 d(k-u)$, while for $u+2 \leqslant j \leqslant k$, it occurs with coefficient $2 d(k-j+1)$. Thus the second term is at most

$$
2 d n(u+1)(k-u)+2 d n(k-u)(k-u-1) / 2=d n(k-u)(k+u+1) .
$$

Adding this to the upper bound for the first term we get at most $d n(k-u)(k+u+4)$ as claimed.

For the following discussion we need a lemma:

Lemma 8.4 (Cost of evaluation of a polynomial at a matrix). Let $M \in \mathbb{F}^{n \times n}$ be a matrix and $f \in \mathbb{F}[x]$ a polynomial with degree $d<n$. Then the evaluation $f(M)$ can be computed using at most $2 d n^{3}$ elementary field operations.

Proof. We take $2 n^{3}$ elementary field operations as an upper bound for a matrix multiplication. The computation of the powers $M^{2}, M^{3}, \ldots, M^{d}$ needs at most $2(d-$ 1) $n^{3}$ elementary field operations. The multiplication, for each $i=1, \ldots, d$, of $M^{i}$ by a coefficient of $f$ and addition of the result to the already computed matrix (the sum of previous terms) needs another $2 d n^{2}$ elementary field operations. Finally, the constant term of $f$ has to be added along the diagonal, which is yet another $n$ elementary field operations. Since $d+1 \leqslant n \leqslant 2 n^{2}$, this is altogether at most $2 d n^{3}$ as claimed.

Of course, this immediately implies:

Corollary 8.5 (Small degree minimal polynomial). If $\operatorname{deg} \mu_{M, \mathbb{F}_{q}^{n}}<n$, then the output of Algorithm 5 can be verified by evaluation using at most

$$
2 \cdot n^{3} \cdot \operatorname{deg} \mu_{M, \mathbb{F}_{q}^{n}}
$$

elementary field operations.

REMARK 8.6. Note that using [1, Theorem 2] we could lower the complexity in Lemma 8.4 to $O\left(\sqrt{d} n^{3}\right)$ provided we stored $O(\sqrt{d})$ matrices in memory at the same time. However, since storing a matrix in $\mathbb{F}^{n \times n}$ needs $O\left(n^{2}\right)$ of memory, this approach would often become impractical before a concrete problem would become intractable because of time constraints. We use our estimates in Lemma 8.4 because of these practical considerations. However, in some practical situations, an improved polynomial evaluation algorithm using more memory may be suitable.

We now present Algorithm 6 that can be run after Algorithm 5 to verify the correctness of the resulting polynomial deterministically. 


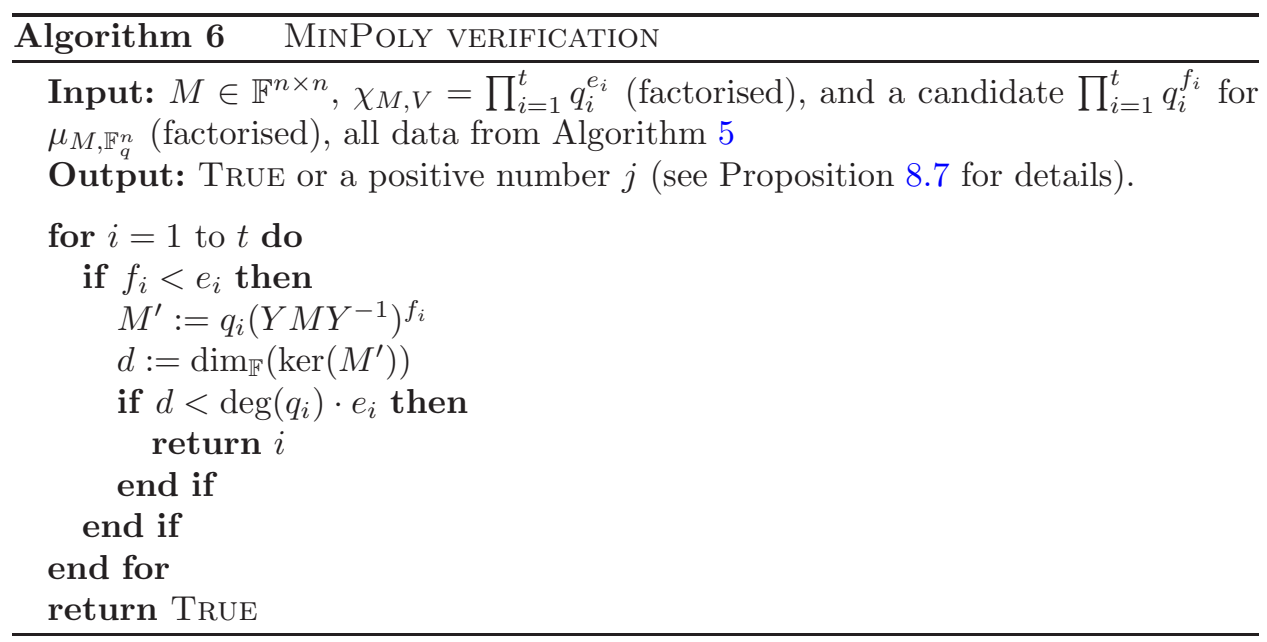

Proposition 8.7 (Deterministic minimal polynomial verification).

If Algorithm 6 is called with candidate minimal polynomial $\prod_{i=1}^{t} q_{i}^{f_{i}}$ from Algorithm 5, then it either returns TRUE or a positive integer $j$. In the former case, $\mu_{M, \mathbb{F}_{q}^{n}}=\prod_{i=1}^{t} q_{i}^{f_{i}}$, while in the latter case the multiplicity of $q_{j}$ in $\mu_{M, \mathbb{F}_{q}^{n}}$ is greater than $f_{j}$. The number of elementary field operations required by Algorithm 6 is at most

$$
n^{3} \cdot \sum_{i=1}^{t}\left(2 \operatorname{deg} q_{i}+2\left\lceil\log f_{i}\right\rceil+1\right) .
$$

Proof. Let $r_{i}:=\operatorname{deg} q_{i}$ for $i=1, \ldots, t$. We again view $\mathbb{F}^{n}$ as $\mathbb{F}[x]$-module as in Section 6 by letting $x$ act as right multiplication by $M$. By [7, Theorem 3.12], it is isomorphic to a direct sum of primary cyclic $\mathbb{F}[x]$-modules

$$
\mathbb{F}^{n} \cong \bigoplus_{i=1}^{t} \bigoplus_{j=1}^{m_{i}} w_{i, j} \mathbb{F}[x]
$$

such that $\operatorname{ord}_{M}\left(w_{i, j}\right)=q_{i}^{f_{i}, j}$ with $e_{i} \geqslant f_{i, 1} \geqslant f_{i, 2} \geqslant \cdots \geqslant f_{i, m_{i}} \geqslant 1$ and $\sum_{j=1}^{m_{i}} f_{i, j}=$ $e_{i}$. Thus, for each $i, q_{i}$ occurs in $\mu_{M, \mathbb{F}_{q}^{n}}$ with multiplicity $f_{i, 1}$, and so in particular $f_{i} \leqslant f_{i, 1}$. The element $q_{i}^{f_{i}}$ acts invertibly on all direct summands $w_{i^{\prime}, j} \mathbb{F}[x]$ with $i^{\prime} \neq i$ since $q_{i}$ is irreducible and every order polynomial of a non-zero vector in such a direct summand is a power of $q_{i^{\prime}}$, by Lemma 4.11. For $i^{\prime}=i$ however, the dimension of the kernel of the action of $q_{i}^{f_{i}}$ on $w_{i, j} \mathbb{F}[x]$ is $r_{i} \cdot \min \left\{f_{i}, f_{i, j}\right\}$. Thus the dimension of the kernel of the action of $q_{i}^{f_{i}}$ on the whole of $\mathbb{F}^{n}$ is equal to

$$
r_{i} \sum_{j=1}^{m_{i}} \min \left\{f_{i}, f_{i, j}\right\} \leqslant r_{i} \sum_{j=1}^{m_{i}} f_{i, j}=r_{i} e_{i}
$$

with equality if and only if $f_{i} \geqslant f_{i, 1}$. Since $f_{i} \leqslant f_{i, 1}$, equality holds above if and only if $f_{i}$ is equal to the multiplicity $f_{i, 1}$ of $q_{i}$ in $\mu_{M, \mathbb{F}_{q}^{n}}$. Therefore, Algorithm 6 always returns the result as stated in the Proposition.

As to the cost, Algorithm 6 evaluates $q_{i}$ at $Y M Y^{-1}$ which needs at most $2 r_{i} n^{3}$ elementary field operations by Lemma 8.4. It then takes the result to the $f_{i}$ th power, 
which can be done by repeated squaring with at most $2 n^{3}\left\lceil\log f_{i}\right\rceil$ elementary field operations, and finally computes the dimension of a null space, which can be done with at most $n^{3}$ elementary field operations (compute a semi echelon basis of the row space of the matrix). Note that we are not using the sparseness of $Y M Y^{-1}$ here.

REMARK 8.8. The cost in Proposition 8.7 is much smaller than $n^{4}$ in many cases. One of the worst cases occurs when $\chi_{M, \mathbb{F}^{n}}$ contains lots of different factors of degree 1 each occurring with multiplicity 3 , and all the $f_{i}$ are equal to 2 . Then Algorithm 6 has to square about $n / 3$ matrices and compute the null spaces of the results. This amounts to about $2 n^{4} / 3$ elementary field operations, which is only about twice as fast as directly evaluating the minimal polynomial at $M$. Note that even in this case only about every sixth entry of $Y M Y^{-1}$ is different from zero.

REMARK 8.9. As in each of our procedures there are some simplifications we could make in practice which do not reduce the worst case complexity estimates. For example, in Algorithm 6, there is no need to compute the kernel of $q_{i}\left(Y M Y^{-1}\right)^{f_{i}}$ if the irreducible $q_{i}$ does not divide any of the relative order polynomials $p^{(j)}$ for $u<j \leqslant k$.

\section{Performance in practice}

In this section we give some experimental evidence concerning the performance of Algorithm 5 in comparison with that of algorithms currently implemented in the GAP library (see [4]).

All computations were done on a machine with an Intel Core 2 Quad CPU Q6600 running at $2.40 \mathrm{GHz}$ with $8 \mathrm{~GB}$ of main memory and two times $4 \mathrm{MB}$ of second level cache.

We were unable to confirm that Magma [2] uses an algorithm based on the canonical forms algorithm of Alan Steel presented in [12] for computing minimal polynomials, although this is indicated in [12, Abstract] and on the web (see http://magma.maths.usyd.edu.au/magma/htmlhelp/text347.htm).

Our colleague Colva Roney-Dougal kindly ran the Baby Monster example matrix $M_{2}$ on Magma and the resulting times were roughly equivalent to the timing in the column "Lib" of Figure 2, suggesting that this is indeed the case. Since the minimal polynomial algorithm in the GAP library is also based on the algorithm in [12], we did not conduct extensive comparison tests of our algorithm on Magma.

\subsection{Guide to the test data}

The timing results are in Figure 2, all times are in seconds. The column marked " $n$ " contains the dimension of the matrix, the column marked " $q$ " the number of elements of the base field. The columns marked "Lib" and "AS" contain the times needed for one run of the minimal polynomial algorithm based on [12] as implemented in the GAP library, and as implemented (by the first author) in the GAP language, respectively. The column "MC" contains the total time for our Monte Carlo algorithm as presented in Algorithm 5. The next three columns marked "Spin", "Fact" and "OrdP" contain the times for the three phases of this algorithm respectively, namely the first phase to compute the characteristic polynomial via relative order polynomials, the second phase to factor all factors of the characteristic polynomial and count multiplicities, and the third phase to compute some absolute order polynomials to guess the minimal polynomial. Finally, the last column marked 
"Ver." contains the time for the deterministic verification via Algorithm 6. The maximal error probability for our Monte Carlo algorithm was $\varepsilon=1 / 100$ for all runs.

\section{2. $\quad$ The test matrices}

Next, we describe the matrices $M_{1}, \ldots, M_{10}$ we used.

(a) The matrices $M_{1}$ and $M_{1}^{\prime}$ were purely random matrices from $\mathbb{F}_{3}^{1000 \times 1000}$ with all entries chosen with uniform distribution from the field $\mathbb{F}_{3}$. Such matrices are with very high probability cyclic, that is, their characteristic and minimal polynomials are equal. Usually, Algorithm 3 only has to pick very few random vectors for such matrices. The for loop of Algorithm 6 quickly checks whether the least common multiple of the relative order polynomials (which is the input candidate polynomial) already has degree $n$. It turned out that $M_{1}^{\prime}$ was cyclic but not $M_{1}$, and this explains the big differences in the runtimes for these matrices.

(b) The matrix $M_{2}$ is one coming from actual applications. Namely, it is the matrix $a+b+a b$ where the two matrices $a, b \in \mathbb{F}_{2}^{4370 \times 4370}$ describe the action of two standard generators of the Baby monster sporadic simple group on its smallest faithful simple module over $\mathbb{F}_{2}$. The matrices $a$ and $b$ were downloaded from the WWW Atlas of group representations (see [16]). The matrix $a+b+a b$ is interesting because it is one of the algebra words that is used in the MEATAxE (see [11] and [6]) to compute composition series of modules and we could very well imagine using the minimal polynomial instead of the characteristic polynomial in some places in the MeAtAxe.

The reason why the standard algorithm for the minimal polynomial performed rather badly on this matrix is that its characteristic polynomial has irreducible factors of degrees $1,1,2,4,6,88,197,854$ and 934 with respective multiplicities $2,2277,4,1,1,1,1,1$ and 1 . Therefore the standard algorithm spins up large subspaces many times.

(c) The matrices $M_{3}-M_{7}$ were constructed in the following way: In the language of $\mathbb{F}[x]$-modules we chose the order polynomials of the generators of their primary cyclic submodules, that is we chose the minimal polynomials on the primary cyclic submodules. For irreducible factors of degree one this amounts to choosing the sizes and numbers of the Jordan blocks occurring in the Jordan normal form of the matrix. After writing down the corresponding normal form of the matrix we conjugated it with a random element of the general linear group to get a dense matrix with the same normal form.

For $M_{3} \in \mathbb{F}_{5}^{600 \times 600}$ we chose one cyclic summand with minimal polynomial $\left(x-\zeta_{5}\right)^{300}$ plus 300 summands with minimal polynomial $x-\zeta_{5}$, where $\zeta_{5} \in \mathbb{F}_{5}$ is a primitive root. This is a typical case in which our Monte Carlo algorithm and the deterministic verification both perform very well in comparison with older techniques. The reason for this is that the high dimensional cyclic subspace is spun up many times in the standard minimal polynomial algorithm as for the matrix $M_{2}$.

For $M_{4} \in \mathbb{F}_{3}^{1200 \times 1200}$ we chose 400 cyclic summands with minimal polynomial $\left(x-\zeta_{3}\right)^{2}$ plus 400 cyclic summands with minimal polynomial $\left(x-\zeta_{3}\right)$, where again $\zeta_{3} \in \mathbb{F}_{3}$ is a primitive root. In contrast with the matrix $M_{3}$, our algorithms performed very well in this case but they were not much faster than the older techniques, since the standard algorithm run on $M_{4}$ does not spin up many large cyclic subspaces. 
Figure 2: Timings for minimal polynomial computation

\begin{tabular}{|c|r|r|r|r|r|r|r|r|r|r|}
\hline $\mathrm{M}$ & $q$ & $n$ & $\mathrm{Lib}$ & $\mathrm{AS}$ & $\mathrm{MC}$ & Spin & Fact & OrdP & Ver. & $\mathrm{k}$ \\
\hline \hline$M_{1}$ & 3 & 1000 & $1.95^{*}$ & 0.65 & 13.7 & 0.33 & 13.3 & 0.05 & 0 & 2 \\
$M_{1}^{\prime}$ & 3 & 1000 & $1.31^{*}$ & 0.68 & 0.32 & 0.32 & 0 & 0 & 0 & 2 \\
$M_{2}$ & 2 & 4370 & 12975 & 3098 & 5.74 & 3.80 & 1.10 & 0.83 & 3.02 & 2212 \\
$M_{3}$ & 5 & 600 & 59.5 & 21.0 & 0.33 & 0.16 & 0.08 & 0.08 & 0.19 & 301 \\
$M_{4}$ & 3 & 1200 & $2.00^{*}$ & 0.45 & 0.44 & 0.38 & 0.06 & 0.01 & 0.06 & 800 \\
$M_{5}$ & 251 & 600 & 2.9 & 3.3 & 3.26 & 2.82 & 0.55 & 0.04 & 0 & 2 \\
$M_{6}$ & 2 & 2391 & 14.6 & 3.3 & 2.25 & 0.91 & 0.18 & 1.15 & 1.02 & 9 \\
$M_{7}$ & 243 & 220 & 0.77 & 0.88 & 0.36 & 0.34 & 0.01 & 0.01 & 0.21 & 7 \\
$M_{8}$ & 17 & 400 & 0.46 & 0.20 & 0.048 & 0.032 & 0.012 & 0.004 & 0.00 & 399 \\
$M_{9}$ & 17 & 400 & 0.26 & 0.20 & 0.23 & 0.23 & 0 & 0 & 0 & 1 \\
$M_{10}$ & 17 & 400 & 0.26 & 0.19 & 0.22 & 0.22 & 0 & 0 & 0 & 1 \\
\hline
\end{tabular}

* averaged over 10 runs

For $M_{5} \in \mathbb{F}_{251}^{600 \times 600}$ we chose 200 different linear factors $x-\alpha$ and for each added one cyclic space with minimal polynomial $(x-\alpha)^{2}$ and one with $x-\alpha$. This example originally was a worst case scenario for our deterministic verification. However, since $k=2$ is quite small, a deterministic verification can be done relatively cheaply as described in Proposition 8.2 and Remark 7.4, even though the integer $u$ in Algorithm 5 is only 1 . The deterministic verification Algorithm 6 ran very slowly (more than 300 seconds) in this example.

For $M_{6} \in \mathbb{F}_{2}^{2391 \times 2391}$ we chose the irreducible polynomial $f(x)=x^{3}+x^{2}+1 \epsilon$ $\mathbb{F}_{2}[x]$ of degree 3 and added cyclic spaces with respective minimal polynomials $f^{400}$, $f^{200}, f^{100}, f^{50}, f^{25}, f^{12}, f^{6}, f^{3}$ and $f$.

For $M_{7} \in \mathbb{F}_{3^{4}}^{220 \times 220}$ we chose an irreducible polynomial $f(x) \in \mathbb{F}_{10}[x]$ of degree 10 and added cyclic spaces with respective minimal polynomials $f^{10}, f, f^{2}, f^{3}, f$, $f^{2}$ and $f^{3}$.

(d) The matrices $M_{8}$ and $M_{9}$ were standard generators of GL $(400,17)$, conjugated by the pseudo-random element $M_{10}$ of the same group. Note that $M_{8}$ had order 16 while $M_{9}$ and $M_{10}$ had very high order and were cyclic matrices. We chose these examples because they may be typical of difficult cases in an application of the minimal polynomial algorithm for computing the projective order of a matrix.

Our algorithm very quickly discovered that the least common multiple of the relative order polynomials was already equal to the characteristic polynomial.

Acknowledgements. We would like to thank an anonymous referee for invaluable suggestions that improved and streamlined the exposition and in particular encouraged us to think about and improve the deterministic verification procedures.

This research forms part of a Discovery Project and Federation Fellowship Grant of the second author funded by the Australian Research Council. 


\section{References}

1. D. Augot and P. CAmion, 'On the computation of minimal polynomials, cyclic vectors, and Frobenius forms', Linear Algebra Appl. 260 (1997) 61-94. 254,273

2. W. Bosma and J. J. CAnnon, Magma, Handbook of Magma Functions, edn 2.14 (2007), http://magma.maths.usyd.edu.au/magma/htmlhelp/MAGMA. htm. 254, 275

3. W. EBerly, 'Asymptotically efficient algorithms for the Frobenius form', Department of Computer Science, University of Calgary Technical Report (2000), http://pages.cpsc.ucalgary.ca/ eberly/Research/publications. php. 254

4. The GAP Group, GAP - Groups, Algorithms, and Programming, version 4.4.10 (2007), http://www.gap-system.org/. 253, 254, 275

5. M. Giesbrecht, 'Nearly optimal algorithms for canonical matrix forms', SIAM J. Comput. 24 (1995) 948-969. 253, 254

6. D. F. Holt and S. Rees, 'Testing modules for irreducibility', J. Austral. Math. Soc. Ser. A 57 (1994) 1-16. 276

7. N. Jacobson, Basic algebra. I (W. H. Freeman \& Co., San Francisco, CA, 1974). 264, 274

8. W. Keller-Gehrig, 'Fast algorithms for the characteristic polynomial', Theoret. Comput. Sci. 36 (1985) 309-317. 253

9. D. E. KNuth, The art of computer programming, vol. 2, Seminumerical algorithms, 3rd edn, Addison-Wesley Series in Computer Science and Information Processing (Addison-Wesley, Reading, MA, 1998). 256

10. E. A. O'Brien, 'Towards effective algorithms for linear groups', Finite geometries, groups, and computation (de Gruyter, Berlin, 2006) 163-190. 252

11. R. A. PARKER, 'The computer calculation of modular characters (the MeatAxe)', Computational group theory, Durham, 1982 (Academic Press, London, 1984) $267-274.276$

12. A. STEEL, 'A new algorithm for the computation of canonical forms of matrices over fields', Computational algebra and number theory, London, 1993, J. Symbolic Comput. 24 (1997) 409-432. 254, 275

13. A. Storjohann, 'An $O\left(n^{3}\right)$ algorithm for the Frobenius normal form', Proceedings of the International Symposium on Symbolic and Algebraic Computation, Rostock, 1998 (ACM, New York, 1998) 101-104 (electronic). 253,254

14. A. Storjohann, 'Deterministic computation of the Frobenius form (extended abstract)', 42nd IEEE Symposium on Foundations of Computer Science, Las Vegas, NV, 2001 (IEEE Computer Society, Los Alamitos, CA, 2001) 368-377. 253, 254

15. J. VOn ZUR GATHEn and J. Gerhard, Modern computer algebra, 2nd edn (Cambridge University Press, Cambridge, 2003), ISBN 0-521-82646-2. 256 
16. R. Wilson, P. Walsh, J. Tripp, I. Suleiman, S. Rogers, R. Parker, S. Norton, S. Nickerson, S. Linton, J. Bray and R. Aвbott, 'The WWW Atlas of Finite Group Representations' (1999), http:// brauer.maths.qmul.ac.uk/Atlas/. 276

Max Neunhöffer neunhoef@mcs.st-and.ac.uk

University of St Andrews, School of Mathematics and Statistics, North Haugh, St Andrews, Fife KY16 9SS, Scotland, United Kingdom

Cheryl E. Praeger praeger@maths.uwa.edu.au

University of Western Australia, School of Mathematics and Statistics (M019), 35 Stirling Highway, Crawley 6009, Western Australia, Australia 